\title{
The Martian surface radiation environment - a comparison of models and MSL/RAD measurements
}

\author{
Daniel Matthiä ${ }^{1, *}$, Bent Ehresmann ${ }^{2}$, Henning Lohf ${ }^{3}$, Jan Köhler ${ }^{3}$, Cary Zeitlin ${ }^{4}$, Jan Appel ${ }^{3}$, Tatsuhiko Sato ${ }^{5}$, \\ Tony Slaba ${ }^{6}$, Cesar Martin ${ }^{3}$, Thomas Berger ${ }^{1}$, Eckart Boehm ${ }^{3}$, Stephan Boettcher ${ }^{3}$, David E. Brinza ${ }^{7}$, \\ Soenke Burmeister ${ }^{3}$, Jingnan Guo ${ }^{3}$, Donald M. Hassler ${ }^{2}$, Arik Posner ${ }^{8}$, Scot C. R. Rafkin ${ }^{2}$, Günther Reitz ${ }^{1}$, \\ John W. Wilson ${ }^{9}$, and Robert F. Wimmer-Schweingruber ${ }^{3}$ \\ 1 German Aerospace Center, Institute of Aerospace Medicine, Linder Höhe, 51147 Cologne, Germany \\ ${ }^{*}$ Corresponding author: Daniel.Matthiae@dlr.de \\ 2 Southwest Research Institute, Space Science and Engineering Division, Boulder, CO 80302, USA \\ 3 Institute of Experimental and Applied Physics, Christian-Albrechts-University, 24118 Kiel, Germany \\ 4 Southwest Research Institute, Space Science and Engineering Division, Durham, NH 03824, USA \\ 5 Japan Atomic Energy Agency, Tokai, Ibaraki 319-1195, Japan \\ 6 NASA Langley Research Center, 2 West Reid St., MS 188E, Hampton, VA 23681, USA \\ 7 Jet Propulsion Laboratory, California Institute of Technology, Pasadena, CA 91109, USA \\ 8 NASA Headquarters, Washington, DC 20546, USA \\ 9 Old Dominion University, Norfolk, VA 23529, USA
}

Received 6 November 2015 / Accepted 14 January 2016

\begin{abstract}
Context: The Radiation Assessment Detector (RAD) on the Mars Science Laboratory (MSL) has been measuring the radiation environment on the surface of Mars since August 6th 2012. MSL-RAD is the first instrument to provide detailed information about charged and neutral particle spectra and dose rates on the Martian surface, and one of the primary objectives of the RAD investigation is to help improve and validate current radiation transport models.

Aims: Applying different numerical transport models with boundary conditions derived from the MSL-RAD environment the goal of this work was to both provide predictions for the particle spectra and the radiation exposure on the Martian surface complementing the RAD sensitive range and, at the same time, validate the results with the experimental data, where applicable. Such validated models can be used to predict dose rates for future manned missions as well as for performing shield optimization studies.

Methods: Several particle transport models (GEANT4, PHITS, HZETRN/OLTARIS) were used to predict the particle flux and the corresponding radiation environment caused by galactic cosmic radiation on Mars. From the calculated particle spectra the dose rates on the surface are estimated.

Results: Calculations of particle spectra and dose rates induced by galactic cosmic radiation on the Martian surface are presented Although good agreement is found in many cases for the different transport codes, GEANT4, PHITS, and HZETRN/OLTARIS, some models still show large, sometimes order of magnitude discrepancies in certain particle spectra. We have found that RAD data is helping to make better choices of input parameters and physical models. Elements of these validated models can be applied to more detailed studies on how the radiation environment is influenced by solar modulation, Martian atmosphere and soil, and changes due to the Martian seasonal pressure cycle. By extending the range of the calculated particle spectra with respect to the experimental data additional information about the radiation environment is gained, and the contribution of different particle species to the dose is estimated.
\end{abstract}

Key words. Mars - Galactic cosmic radiation - Radiation environment - Human exploration - Numerical models - Exposure

\section{Introduction}

The Mars Science Laboratory (Grotzinger et al. 2012) - Radiation Assessment Detector (MSL-RAD; Hassler et al. 2012) detector on board the Curiosity rover has been the first instrument to measure particle spectra and dose rates both during the cruise to Mars (Zeitlin et al. 2013; Guo et al. 2015; Köhler et al. 2015) and on the Martian surface (Ehresmann et al. 2014; Hassler et al. 2014; Köhler et al. 2014; Rafkin et al. 2014). Besides the continuous exposure to galactic cosmic radiation (GCR), the MSL-RAD instrument has measured several moderate solar energetic particle events during the cruise phase, from December 2011 to July 2012, and a few small solar particle events on the Martian surface since August 2012. During cruise, these events contributed approximately $5 \%$ to the total dose equivalents (Zeitlin et al. 2013), and since arriving at Mars, the contribution of the events to the observed total dose has been small (Hassler et al. 2014). In this paper, we therefore focus on the GCR spectra and their contribution to total radiation dose and dose equivalent. For the determination of the radiation exposure for different mission scenarios and the mitigation of the radiation risk of astronauts during such missions, numerical models are of great value as they allow, for instance, optimization of shielding conditions or selection of appropriate launching schedules (Cucinotta et al. 2010, 2011). Additionally, models are not only helpful for 
prospective mission analysis but are also able to extend and complement the measurements that were performed and thereby broaden our understanding of the radiation field by, for instance, extending the energy range of the particle fluxes or providing estimates of particle fluxes and dose rates for arbitrary atmospheric, or human fabricated shielding scenarios as well as for different solar modulation. Comparing measured particle fluxes, especially neutrons, with model predictions may also provide information about the composition of the atmosphere and soil of Mars.

In order to estimate the health risk of astronauts it is of great importance to have detailed information about the particle spectra. The dose concept commonly used in radiation protection on Earth might not describe adequately the biological effects of irradiation with heavy ions (National Research Council 1967) as encountered in space and on lightly shielded planetary surfaces. Even the quality factor, $Q$, used to describe the increased biological effectiveness of high charge and energy ions as compared to gamma rays, may not adequately describe carcinogenesis risk or impacts on the central nervous system and other degenerative tissue effects. It is therefore essential to know the energy spectra of each particle type as accurately as possible in order to apply suitable radiation risk concepts.

As stated above, during low solar activity (solar minimum) galactic cosmic radiation and secondary particles created by them are the main contributors to the radiation environment on the surface of Mars (Dartnell et al. 2007; Ehresmann 2012) and in the present work we focus on this aspect and do not discuss the influence of solar energetic particle events which will be covered in future work. Several numerical models exist that can calculate the transport of primary nuclei from the GCR through the Martian atmosphere and the backscattering from the soil. The following models are investigated in this work: HZETRN/OLTARIS (Wilson et al. 1991; Slaba et al. 2010a, 2010b, 2013; Singleterry et al. 2011; Norman et al. 2013), GEANT4/PLANETOCOSMICS (Agostinelli et al. 2003; Allison et al. 2006), and PHITS (Niita et al. 2006; Sato et al. 2013). These transport models, which are discussed in more detail below, have to be combined with models of the primary GCR spectra taking into account the solar modulation. Following recent studies evaluating different GCR models concerning their applicability for the calculation of radiation exposure (Mrigakshi et al. 2012, 2013b; Slaba \& Blattnig 2014) the Badhwar-O'Neill 2010 model (O’Neill 2010), named BO-10 hereafter, and the model by Matthiä et al. (2013), named DLR model hereafter (Deutsches Zentrum für Luft- und Raumfahrt, DLR), are used in this work.

The MSL-RAD instrument is, among other things, capable of measuring gamma and neutron spectra (Köhler et al. 2014) and several types of charged particles (Ehresmann et al. 2014). The published results of the first 200 sols (1 solar day, sol, on Mars is about $24 \mathrm{~h}$ and 39 min long) on the Martian surface (between August 2012 and January 2013) are used in this work for a validation of the different numerical radiation transport models. While several publications exist in the literature in which different simulation models and tools have been used to estimate the radiation exposure in interplanetary space during a cruise to Mars (e.g. McKenna-Lawlor et al. 2012b), in the atmosphere and on the surface of Mars (e.g. Simonsen et al. 1990; Townsend et al. 2011; Kim et al. 2014; Gronoff et al. 2015), few data are available on predicted particle spectra. However, comparing such data with measurements gives deeper insight into the accuracy of the modeling of the underlying physical processes. Dose rate comparisons, on the other hand, may be misleading because the dose rate is obtained by integrating over different particle species and wide energy ranges. Thus, agreement in dose rate of model predictions and measurements for a certain scenario is less valuable for model validation in a given exposure scenario than is agreement in energy-dependent particle flux. In this work we make use of data provided by MSL-RAD to benchmark the transport models for the prediction accuracy of secondary particle fluxes as well as resulting dose rates.

\section{Materials and methods}

\subsection{The MSL-RAD measurements on the Martian surface}

The Radiation Assessment Detector (RAD) has been conducting the first-ever radiation measurements on the Martian surface as part of NASA's Mars Science Laboratory (MSL) mission. RAD has been measuring almost continuously since the successful landing of the Curiosity rover in Gale crater on 6 August 2012, for large parts operating in a $100 \%$ duty cycle with a 16 - or 32 -minute operating cadence.

The time frame considered for this research spans from 18 August 2012 to 30 January 2013, or from sol 13 to 173 in MSL terminology. We compare the presented simulation results with particle spectra measured by RAD during this time period as published in Ehresmann et al. (2014) and Köhler et al. (2014). Measurements made by the Rover Environmental Monitoring Station (REMS; Gómez-Elvira et al. 2014) show that the average atmospheric column depth above the rover in this time frame was $\sim 21-22 \mathrm{~g} / \mathrm{cm}^{2}$ (Haberle et al. 2014). In this work we used an atmospheric depth of $22 \mathrm{~g} / \mathrm{cm}^{2}$ for our simulation setup.

\subsubsection{The RAD sensor head}

The RAD sensor head (RSH, a schematic is shown in Fig. 1) contains a stack of detectors for charged and neutral particle measurements: three Si detectors (named A, B, and C), a CsI scintillator (D), and two plastic scintillators ( $\mathrm{E}$ and $\mathrm{F}$ ). A detailed description of the RSH can be found in Hassler et al. (2012).

\subsubsection{Measurements of particle spectra, dose, and LET}

Neutral particles are measured in $\mathrm{D}$ and $\mathrm{E}$, with $\mathrm{C}$ and $\mathrm{F}$ acting as anticoincidence against charged particles (see Köhler et al. 2014 for details of the neutral particle measurements). Using an inversion technique, we are able to derive separate spectra for $\gamma$-rays and neutrons, as both $\mathrm{D}$ and $\mathrm{E}$ have different sensitivities for those particle types.

For purposes of charged particle detection all detectors are used (a description of the charged particle measurement process is given by Ehresmann et al. 2014). Here, A and B act as a telescope defining the acceptance cone for incoming charged particles. RAD analysis distinguishes between two types of charged particles: particles penetrating the RSH and those that stop in either one of detectors B, C, D, or E. Stopping particles can be resolved as energy- and particle-typedependent spectra, while integral fluxes are derived for the different species of penetrating particles. The full opening angle for stopping particles is $\sim 60^{\circ}$ from the upper hemisphere, while particles penetrating the RSH have a full acceptance angle of $36^{\circ}$ from both upper and lower hemisphere, although the fraction of penetrating particles coming from the lower half, the Martian soil, is estimated to be almost negligibly small (McKenna-Lawlor et al. 2012a).

RAD measures the absorbed radiation dose in two detectors: B (made of silicon) and E (tissue-equivalent plastic), both with a $4 \pi$ acceptance angle. To derive the biologically-relevant dose equivalent from the absorbed dose, the typical approach is to use the average quality factor $\langle Q\rangle$ of the incoming radiation for conversion. As described by Zeitlin et al. (2013), the quality factor is dependent on 


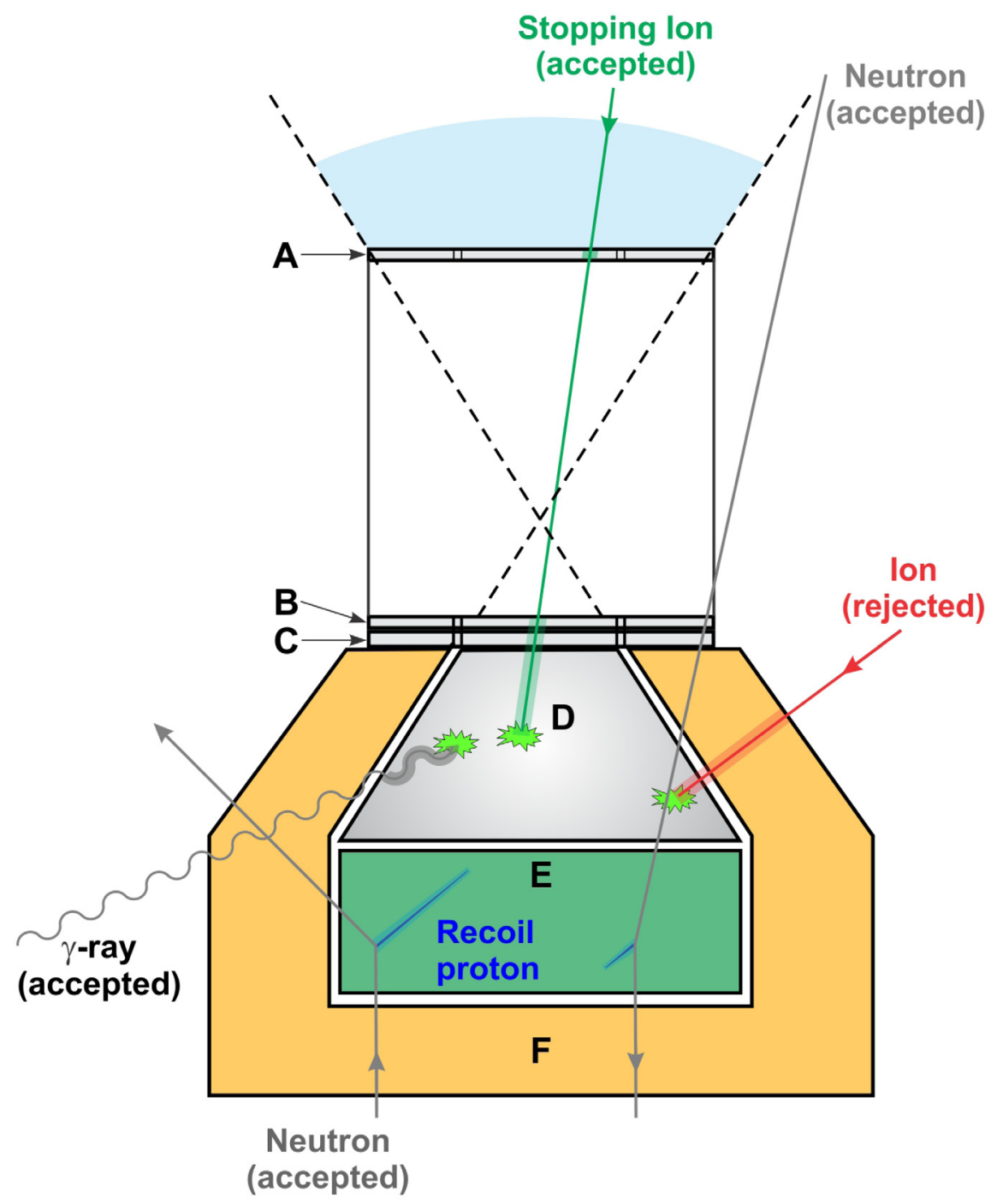

Fig. 1. Schematic of the RAD sensor head (from Köhler et al. 2014, cf. also Posner et al. 2005).

the linear energy transfer (LET) spectrum of the radiation, and thus the average $\langle Q\rangle$ can be obtained by knowledge of this LET spectrum. RAD measures the LET in silicon in detector B in coincidence with detector A to limit the possible path length of the particles in the detector; the LET in silicon is then scaled to (approximate) LET in water for purposes of the quality factor calculation.

\subsection{Galactic cosmic ray model}

The main source of ionizing radiation in deep space is galactic cosmic radiation and, although MSL-RAD has measured several solar energetic particle events during its cruise phase and on the Martian surface, the present work is limited to investigations of the GCR induced radiation. Mrigakshi et al. (2012, 2013a, 2013b) and Slaba et al. (2014) have investigated several models suitable as input for the calculation of radiation exposure in space and found that the most suitable models currently available are BO-10 model (O'Neill 2010) and the DLR model (Matthiä et al. 2013). GCR consists mainly of fully ionized atomic nuclei of which about $87 \%$ are hydrogen, $12 \%$ helium, and about 1\% heavier nuclei (Simpson 1983). There is a slight dependence of the relative abundances on the solar cycle. Despite their comparably low intensity, heavy ions have a great impact on the radiation exposure in space due to their high biological relevance (Durante \& Cucinotta 2008). In general, the contributions of primary nuclei up to atomic numbers of $Z=26(\mathrm{Fe})$ are sufficient to estimate the radiation exposure to humans from GCR. Iron in particular is of great importance for the dose equivalent due to its high biological relevance in combination with its relatively high abundance (Simpson 1983). In this work, contributions of all nuclei from hydrogen up to iron were considered.

Figure 2 illustrates the primary GCR spectra of hydrogen $(Z=1)$, helium $(Z=2)$, carbon $(Z=6)$, and iron $(Z=26)$ as described by the two models used in this work: BO-10 and DLR. In order to benchmark the primary GCR models and the transport of particles through the Martian atmosphere the solar modulation for the time period of measurements (August 2012-January 2013) was considered in the GCR models. The modulation parameter $\phi$ during this period was between $533 \mathrm{MV}$ and $643 \mathrm{MV}$ (cf. http://cosmicrays.oulu.fi/phi/ Phi_mon.txt). Although Mrigakshi et al. (2013a) found differences of more than $20 \%$ in the estimated dose rates comparing the two GCR models during solar minimum periods, the primary spectra for the time of the MSL-RAD measurements considered here are very similar. Calculations of particle spectra and radiation exposure with PHITS and GEANT4 performed in this work showed that the differences arising from the application of the two GCR models for this period of solar modulation are small, yielding differences in the dose rates on the order of a few percent. The results shown below are limited to the results using the DLR model in case of PHITS and GEANT4, and to the BO-10 model in case of HZETRN/ OLTARIS. 


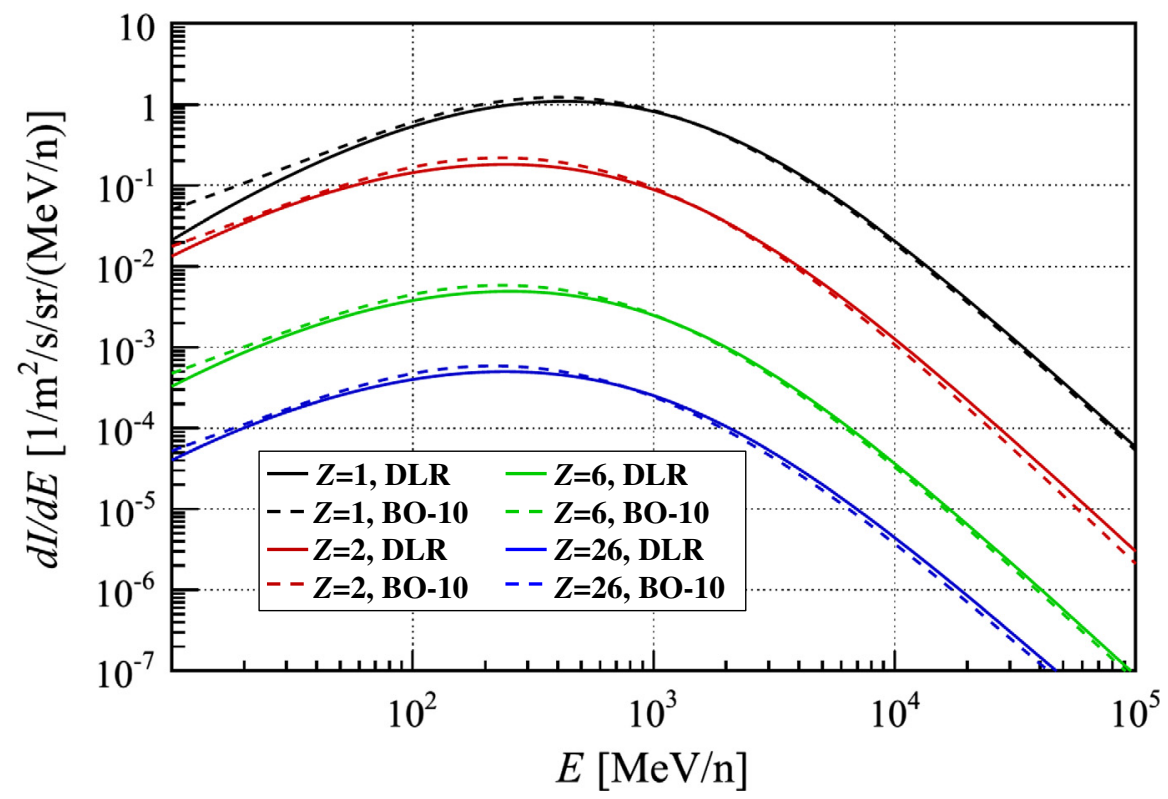

Fig. 2. Primary GCR spectra, exemplarily for hydrogen $(Z=1)$, helium $(Z=2)$, carbon $(Z=6)$, and iron $(Z=26)$, for the time between August 2012 and January 2013 as described by the DLR (Matthiä et al. 2013) and Badhwar/O'Neill 2010 (BO-10) (O’Neill 2010) models. Calculations were performed taking into account all primary nuclei from hydrogen to iron.

\subsection{Martian soil and atmosphere}

The atmospheric composition used in the GEANT4 and HZETRN/ OLTARIS simulations is based on the Mars Global Reference Atmospheric Model 2001 (Mars-GRAM 2001) (Justus \& Johnson 2001; Justus et al. 2006), which is an engineering-level atmospheric model. It utilizes Mars Global Surveyor's Mars Orbiter Laser Altimeter (MOLA; Smith et al. 2001) for surface topography. For atmospheric properties up to $80 \mathrm{~km}$ height, it relies on the NASA Ames Mars General Circulation model (MGCM; Haberle et al. 1993). Above $80 \mathrm{~km}$ it uses the Mars Thermospheric General Circulation Model (MTGCM; Bougher et al. 1990).

The Mars-GRAM 2001 atmospheric model consists of multiple layers with different mass densities. Each layer consists of 95.7 mass $\% \mathrm{CO}_{2}, 2.7$ mass \% N, and 1.6 mass \% Ar. Starting at a total atmospheric height of $90 \mathrm{~km}$, we set the soil and detector level at the surface to a depth of $22 \mathrm{~g} / \mathrm{cm}^{2}$, corresponding to the mean atmospheric column density within the comparison time frame from DOY 232 2012 to DOY 482013 at the MSL rover. The mean pressure data is derived from the Mars Climate Database (MCD 5.0). For PHITS a pure $\mathrm{CO}_{2}$ atmosphere with the same depth was used. Calculations performed with GEANT4 using the two atmospheric compositions did not result in a significant change in secondary particle intensities and dose rate.

The Martian soil composition is modeled based on the regolith definition in OLTARIS. It has a density of $1.7 \mathrm{~g} / \mathrm{cm}^{3}$ with molecular percentages given in Table 1. For all simulations the soil thickness was at least $20 \mathrm{~m}$.

\subsection{Transport environments}

\subsubsection{GEANT4/PLANETOCOSMICS}

GEANT4 (GEometry And Tracking) (Agostinelli et al. 2003; Allison et al. 2006) is a Monte-Carlo method based toolkit capable of calculating the transport of particles through matter. PLANETOCOSMICS (http://cosray.unibe.ch/laurent/planetocosmics/) is a GEANT4 application providing geometries and descriptions of planetary atmospheres, soils, and magnetospheres, for instance for Earth and Mars, and can be used to calculate the transport of arbitrary primary particles in and through these planetary environments and the creation of secondary
Table 1. Martian soil composition from OLTARIS and used in the simulations (soil density $1.7 \mathrm{~g} / \mathrm{cm}^{3}$ ).

\begin{tabular}{lcccc}
\hline \hline & $\mathrm{SiO}_{2}$ & $\mathrm{Fe}_{2} \mathrm{O}_{3}$ & $\mathrm{Al}_{2} \mathrm{CaK}_{2} \mathrm{MgNa}_{2} \mathrm{O}_{7}$ & $\mathrm{H}_{2} \mathrm{O}$ \\
\hline $\begin{array}{l}\text { Molecular } \\
\text { percentage }\end{array}$ & 51.2 & 9.3 & 32.1 & 7.4 \\
\hline
\end{tabular}

particles. To obtain the results presented in this work GEANT4 version 10.p02 was used. GEANT4 provides a large number of so-called physics lists describing the interactions of particles with matter. One of the goals of this work is to investigate the applicability of different physics lists for the calculation of secondary particle spectra and dose rates on the Martian surface or on planetary surfaces in general. In the lower part of Table 2 an overview of the different selections of physics lists in GEANT4 is given. These lists were selected according to the recommendations given by the GEANT4 community for such an application. The different lists are mainly related to inelastic hadronic (nucleon or nuclear) interactions. For electromagnetic interactions the most precise standard list (emstandard_opt3) was selected. The application of an advanced list for electromagnetic interactions (emstandard_opt4) for one selected hadronic list did not affect the resulting dose rates or particle spectra. Comparison with these RAD data has resulted in improved selection of physics lists, and the differences arising from the selection of physics lists are discussed below. For the calculation with GEANT4 primary nuclei from hydrogen $(Z=1)$ to nickel were used $(Z=28)$.

\subsubsection{HZETRN/OLTARIS}

HZETRN (Wilson et al. 1991; Slaba et al. 2010a, 2010b, 2013; Norman et al. 2013) is a deterministic transport code providing numerical solutions to the time-independent, linear Boltzmann equation (Wilson et al. 1991). The transport formalism allows for a converging sequence of physical approximations to be considered, allowing highly efficient computational procedures to be implemented. Typical run times for full GCR calculations range from seconds to minutes on a single CPU. The version of the code used herein utilizes a bidirectional transport approach for neutrons and light ions (Slaba et al. 2010a), allowing backscattered albedo neutron contributions to be described in the present comparisons. Heavier ions are treated within the 
Table 2. Dose rates measured by MSL-RAD on the surface of Mars and calculated values. Values in parentheses are the dose rates and the derived quality factor for a restricted zenith angle $\theta<30^{\circ}$.

\begin{tabular}{|c|c|c|c|c|c|c|c|c|c|}
\hline & \multirow{2}{*}{$\begin{array}{c}\text { MSL-RAD } \\
\text { (Hassler et al. 2014) }\end{array}$} & \multicolumn{5}{|c|}{ GEANT4.10.p02 } & \multirow[t]{2}{*}{ PHITS } & \multirow[t]{2}{*}{ OLTARIS2013 } & \multirow{2}{*}{$\begin{array}{l}\text { HZETRN/ } \\
\text { OLTARIS }\end{array}$} \\
\hline & & {$[1]$} & {$[2]$} & {$[3]$} & {$[4]$} & {$[5]$} & & & \\
\hline dose rate in silicon $[\mathrm{mGy}=\mathrm{d}]$ & & $0.16(0.017)$ & $0.15(0.17)$ & $0.15(0.017)$ & $0.15(0.016)$ & $0.16(0.017)$ & $0.15(0.019)$ & 0.13 & $0.14(0.019)$ \\
\hline dose rate in tissue $[\mathrm{mGy}=\mathrm{d}]$ & $0.21 \pm 0.04$ & $0.19(0.021)$ & $0.19(0.021)$ & $0.19(0.020)$ & $0.18(0.020)$ & $0.2(0.021)$ & $0.20(0.024)$ & 0.16 & $0.18(0.024)$ \\
\hline dose equivalent rate $[\mathrm{mSv}=\mathrm{d}]$ & $0.64 \pm 0.12$ & $0.52(0.063)$ & $0.51(0.063)$ & $0.51(0.067)$ & $0.51(0.069)$ & $0.61(0.068)$ & $0.6(0.081)$ & 0.52 & $0.54(0.076)$ \\
\hline Quality factor & $3.0 \pm 50.26$ & $2.7(3.0)$ & $2.7(3.1)$ & $2.7(3.3)$ & $2.8(3.5)$ & $3.0(3.2)$ & $3.0(3.4)$ & 3.2 & $3.0(3.2)$ \\
\hline
\end{tabular}

GEANT4 physics list setups

[1]

$[2]$

Physics Lists: emstandard_opt3, G4HadronPhysicsINCLXX, G4IonINCLXX

\section{Models:}

Ions: INCL v5.1.14.2 $(0 \mathrm{eV}<E<54 \mathrm{GeV})$; FTFP $(53.9 \mathrm{GeV}<E<1 \mathrm{TeV})$

Neutrons/Protons: PRECO $(0 \mathrm{eV}<E<2 \mathrm{MeV})$; INCL v5.1.14.2 (1 MeV $<E<3 \mathrm{GeV})$; Bertini $(2.9 \mathrm{GeV}<E<9.9 \mathrm{GeV})$; QGSP $(12 \mathrm{GeV}<E<100$ $\mathrm{TeV})$; FTFP $(9.5 \mathrm{GeV}<E<25 \mathrm{GeV})$

Physics Lists: emstandard_opt3, user defined

\section{Models:}

Ions: INCL v5.1.14.2 $(0 \mathrm{eV}<E<48 \mathrm{GeV})$; FTFP $(47.999 \mathrm{GeV}<E<1 \mathrm{TeV})$

Protons: PRECO $(0 \mathrm{eV}<E<2 \mathrm{MeV})$; INCL v5.1.14.2 $(1 \mathrm{MeV}<E<3 \mathrm{GeV})$; Binary Cascade $(2.9 \mathrm{GeV}<E<9.9 \mathrm{GeV})$; QGSP $(12 \mathrm{GeV}<E<100$

TeV); FTFP $(9.5 \mathrm{GeV}<E<25 \mathrm{GeV})$

Neutrons: NeutronHPInelastic $(0 \mathrm{eV}<E<20 \mathrm{MeV})$; INCL v5.1.14.2 (19.9 MeV $<E<3 \mathrm{GeV})$; Binary Cascade $(2.9 \mathrm{GeV}<E<9.9 \mathrm{GeV})$; QGSP $(12 \mathrm{GeV}<E<100 \mathrm{TeV})$; FTFP $(9.5 \mathrm{GeV}<E<25 \mathrm{GeV})$

Physics Lists: emstandard_opt3, G4HadronPhysicsQGSP_BIC_HP, G4IonPhysics

\section{Models:}

Ions: Binary Light Ion Cascade $(0 \mathrm{eV}<E<4 \mathrm{GeV})$; FTFP $(2 \mathrm{GeV}<E<100 \mathrm{TeV})$

Protons: Binary Cascade $(0 \mathrm{eV}<E<9.9 \mathrm{GeV})$; QGSP $(12 \mathrm{GeV}<E<100 \mathrm{TeV})$; FTFP $(9.5 \mathrm{GeV}<E<25 \mathrm{GeV})$

Neutrons: NeutronHPInelastic $(0 \mathrm{eV}<E<20 \mathrm{MeV})$; Binary Cascade $(19.9 \mathrm{MeV}<E<9.9 \mathrm{GeV})$; QGSP $(12 \mathrm{GeV}<E<100 \mathrm{TeV})$; FTFP $(9.5 \mathrm{GeV}<E<25 \mathrm{GeV})$

Physics Lists: emstandard_opt3, G4HadronPhysicsQGSP_BIC_HP, G4IonQMDPhysics

\section{Models:}

Ions: QMDModel $(0 \mathrm{eV}<E<10 \mathrm{TeV})$

Protons: Binary Cascade $(0 \mathrm{eV}<E<9.9 \mathrm{GeV})$; QGSP $(12 \mathrm{GeV}<E<100 \mathrm{TeV})$; FTFP $(9.5 \mathrm{GeV}<E<25 \mathrm{GeV})$

Neutrons: NeutronHPInelastic $(0 \mathrm{eV}<E<20 \mathrm{MeV})$; Binary Cascade $(19.9 \mathrm{MeV}<E<9.9 \mathrm{GeV})$; QGSP $(12 \mathrm{GeV}<E<100 \mathrm{TeV})$; FTFP $(9.5 \mathrm{GeV}<E<25 \mathrm{GeV})$

Physics Lists: emstandard_opt3, G4HadronPhysicsQGSP_BERT_HP, G4IonINCLXX

Models:

Ions: INCL v5.1.14.2 $(0 \mathrm{eV}<E<54 \mathrm{GeV})$; FTFP $(53.9 \mathrm{GeV}<E<1 \mathrm{TeV})$

Protons: BertiniCascade $(0 \mathrm{eV}<E<9.9 \mathrm{GeV})$; QGSP $(12 \mathrm{GeV}<E<100 \mathrm{TeV})$; FTFP $(9.5 \mathrm{GeV}<E<25 \mathrm{GeV})$

Neutrons: NeutronHPInelastic $(0 \mathrm{eV}<E<20 \mathrm{MeV})$; BertiniCascade $(19.9 \mathrm{MeV}<E<9.9 \mathrm{GeV})$; QGSP $(12 \mathrm{GeV}<E<100 \mathrm{TeV})$; FTFP $(9.5 \mathrm{GeV}<E<25 \mathrm{GeV})$ 
(a)

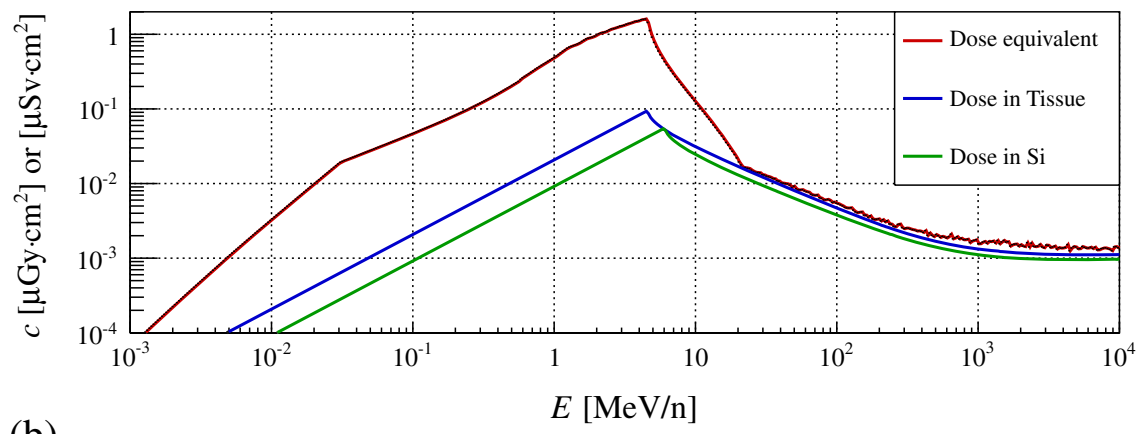

(b)

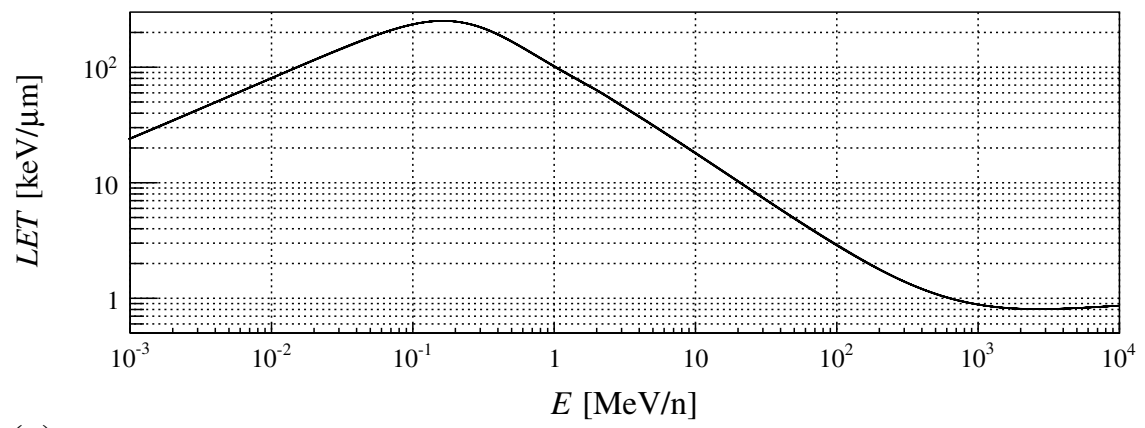

(c)

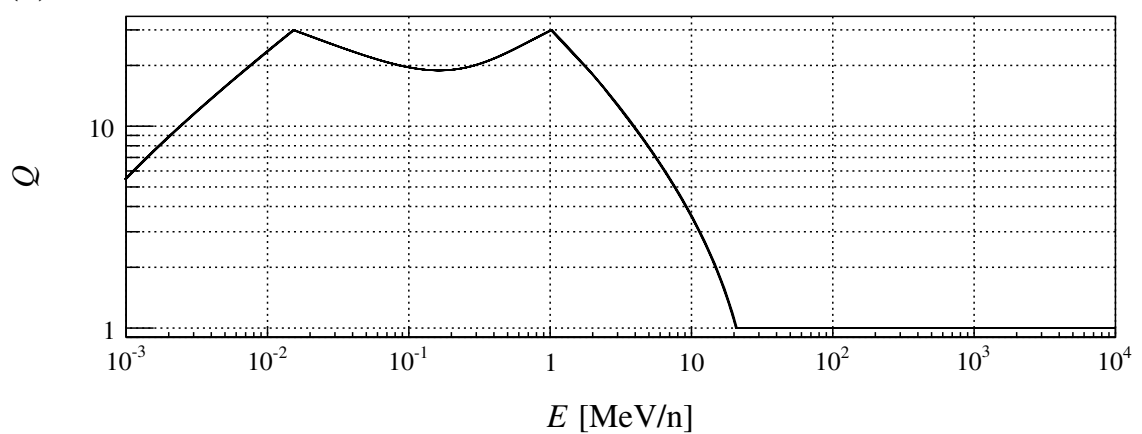

Fig. 3. Exemplary fluence-to-dose conversion factors for alpha particles versus particle energy (a). The quality factor $Q$ (c) is calculated from the energy-dependent linear energy transfer (LET, b) following the ICRP definition of the Q(LET) dependence (ICRP 2007).

straight-ahead approximation (Wilson et al. 1991). The pion, muon, and electromagnetic cascade components (Norman et al. 2013) are also included here. Recent improvements to the code (Wilson et al. 2014, 2015) include 3D corrections for neutrons and light ions which were not included here but may be considered in future work. The NUCFRG3 (Adamczyk et al. 2012) model is used for describing nuclear fragmentation of heavy ions. Light ion and neutron interaction models are described elsewhere (Wilson et al. 1991; Cucinotta 1993; Cucinotta et al. 1996). The implementation of HZETRN for Mars surface calculations considers the atmospheric geometry and variable density profile in a ray-by-ray computational procedure as described by Slaba et al. (2013).

For the calculations reported here, we used data from the Oulu neutron monitor (Usoskin et al. 2011) to determine a linear relation between neutron monitor count rates and $\varphi$ for 2012. This procedure is valid provided that the range of count rates is small, as it was in the period considered. The relationship was applied to Oulu neutron monitor count rate data from 2013 to determine monthly $\varphi$ values, so that we have a consistent set of values for the duration of the MSL mission from launch to present. Averaging over sols 13-173, we find an average $\varphi$ value of $577 \mathrm{MV}$. The results generated from HZETRN presented herein can also now be generated with the "On-Line Tool for the Assessment of Radiation in Space" (OLTARIS; Singleterry et al. 2011), and are labeled as HZETRN/OLTARIS in the following plots and tables. The only exceptions are the photon, pion, muon, electron, and positron results which are not yet available through the website.

In addition, we also show earlier results from the OLTARIS framework which were presented in Ehresmann et al. (2014) and obtained in 2013. These results are labeled as OLTARIS2013.

An important difference between the OLTARIS2013 and HZETRN/OLTARIS results presented herein is the geometry setup used in the OLTARIS2013 calculations. In those earlier calculations, the Martian geometry was represented by a $21 \mathrm{~g} / \mathrm{cm}^{2}$ sphere of $\mathrm{CO}_{2}$. Albedo particles were not included, and large off-vertical atmosphere thicknesses were not represented. The HZETRN/OLTARIS results, on the other hand, account for albedo neutrons and longer off-vertical atmospheric thicknesses in the ray-by-ray transport procedure, as described by Slaba et al. (2013). The comparison to the results from 2013 is performed in order to illustrate both the development of the code since the publication of the first charged particle spectra measured on the Martian surface (Ehresmann et al. 2014) and the limitations of the simplified geometry in the early calculations $\left(21 \mathrm{~g} / \mathrm{cm}^{2}\right.$ sphere of $\mathrm{CO}_{2}$ ).

\subsubsection{Particle and Heavy Ion Transport code System (PHITS)}

PHITS (Particle and Heavy Ion Transport code System; Niita et al. 2006; Sato et al. 2013) is a Monte-Carlo code for calculating the 
(a)
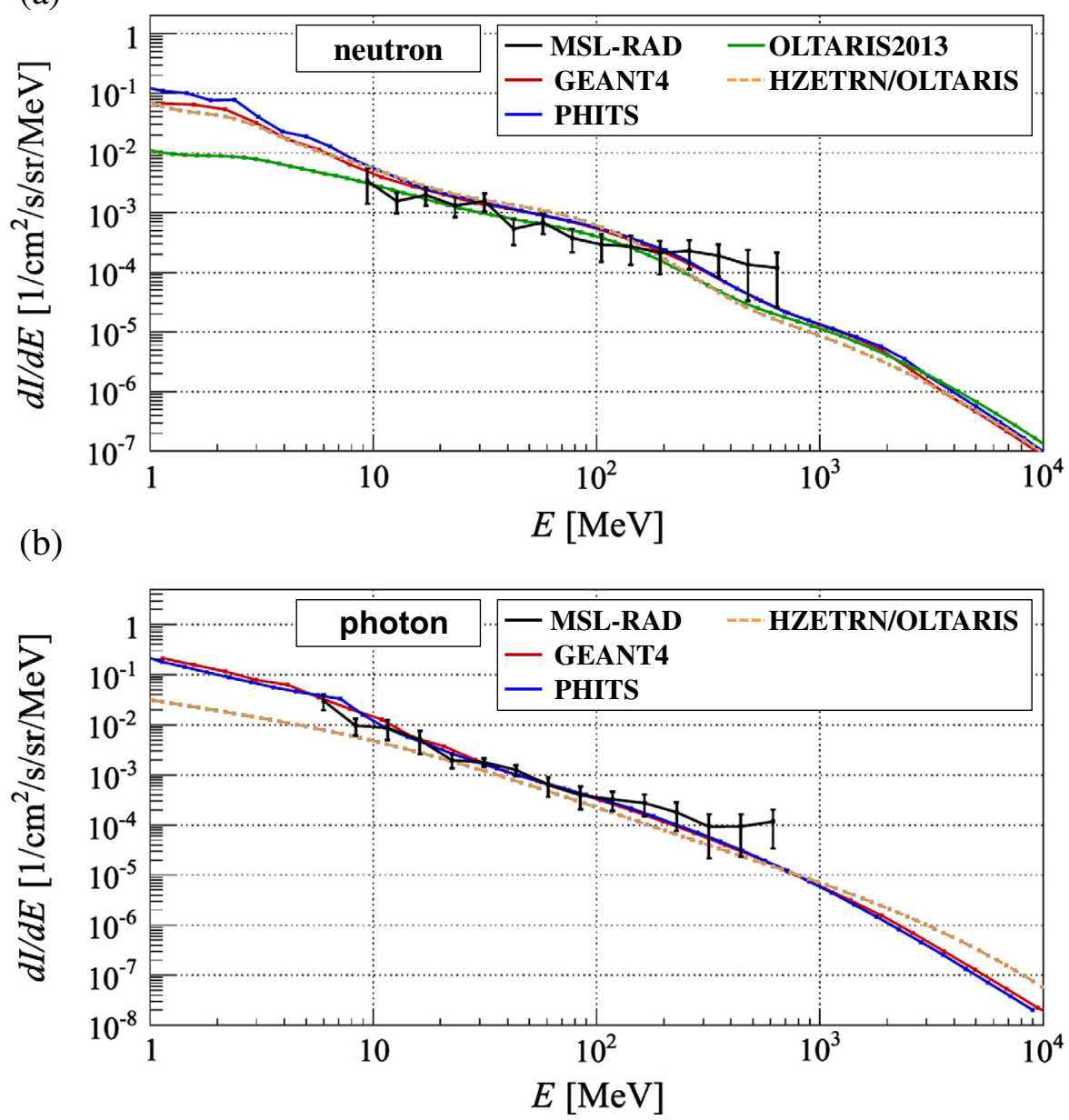

Fig. 4. Neutral particle spectra (all incident angles) on the Martian surface measured between 19 August 2012 and 17 February 2013 by MSLRAD (black, Köhler et al. 2014) and calculated for the same period by different simulation tools: GEANT4 (red), PHITS (blue), OLTARIS2013 (green), HZETRN/OLTARIS (ocher).

transport of particles through matter. PHITS version 2.76 was used in this study. The intranuclear cascade models JAM (Nara et al. 1999) and INCL4.6 (Boudard et al. 2013) were employed for simulating nucleon-nucleus interactions above and below $3 \mathrm{GeV}$, respectively, except for neutron interactions below $20 \mathrm{MeV}$, where the nuclear data library JENDL-4.0 (Shibata et al. 2011) coupled with the event generator mode version 2.0 (Ogawa et al. 2014) was adopted in the simulation. The total nucleon-nucleus interaction cross-sections were calculated by a model specially adjusted for high-energy particle transport simulations (Sato et al. 2014). An updated version of the quantum molecular dynamics model, JQMD (Niita et al. 1995), was used for simulating nucleus-nucleus interactions. The charge and energy of primary particles were determined using the same model employed in the GEANT4 simulation. In the PHITS simulation, all primary and secondary particles were traced down to $1 \mathrm{MeV}$, except for neutrons, electrons, positrons, and photons, where the cut-off energy of neutrons was set to $0.1 \mathrm{meV}$ and that for the others to $10 \mathrm{keV}$.

\subsection{Calculation of dose rates}

Particle spectra calculated with the different transport codes and primary input spectra were used to estimate the radiation exposure on Martian ground. The results were compared to the measurements of MSL-RAD. In order to convert the particle fluxes on ground to the dose rates, pre-calculated fluence-to-dose conversion factors were used. These conversion factors were the same for all transport codes which means that differences in the calculated dose rates originate in the transport models only. GEANT4 was used to calculate the conversion factors for different dose quantities: absorbed dose in silicon, absorbed dose in tissue, and dose equivalent in tissue. Figure 3a shows the conversion factors from fluence to dose in tissue and in silicon and to dose equivalent exemplarily for $\alpha$-particles. The dose equivalent can be calculated by multiplying the dose in tissue with the quality factor $Q$ (Fig. 3c) defined by the ICRP (ICRP 1991, 2007). $Q$ is a function of the unrestricted LET in water (Fig. 3b). The quality factor is used to weight the energy deposition with the biological impact which is LET dependent.

\section{Results}

\subsection{Neutral particle spectra}

Figure 4 shows the calculated and measured neutron (4a) and photon fluxes (4b). The results of GEANT4 that are shown here were obtained with physics list setup [1] from Table 2. The selection of this physics list was guided by comparison with the RAD data. The measured data is taken from Köhler et al. (2014). The results of the PHITS, HZETRN/OLTARIS, and GEANT4 simulations agree reasonably well with each other over the whole energy range for both neutrons and photons. Apart from setup [2] from Table 2 the selection of the physics list in GEANT4 had no significant influence on the predicted neutron flux. Using the Bertini cascade model, as in setup [2], however, greatly enhances the calculated neutron 


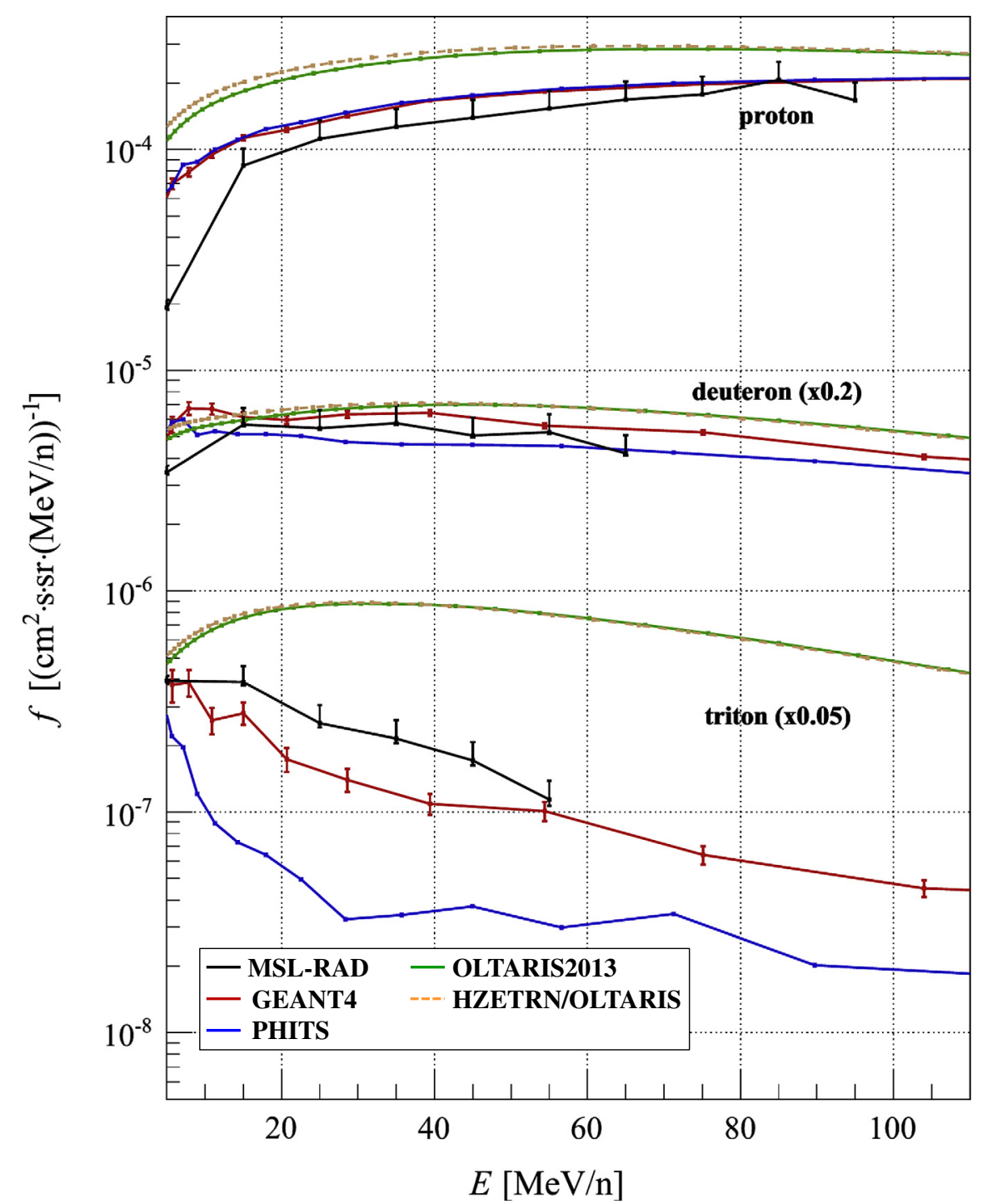

Fig. 5. Hydrogen isotope spectra (from top to bottom: proton, deuteron, and triton) for zenith angles smaller than $30^{\circ}$ on the Martian surface measured between 19 August 2012 and 17 February 2013 by MSL-RAD (black, Ehresmann et al. 2014) and calculated for the same period by different simulation tools: GEANT4 (red), PHITS (blue), OLTARIS2013 (green), ZETRN/OLTARIS (ocher). The data have been scaled by the factors given in parentheses.

fluxes in GEANT4. The OLTARIS2013 results tend to underestimate all other codes below $100 \mathrm{MeV}$ due to the simplified representation of the mars geometry and lack of albedo contributions.

In Figure $4 \mathrm{~b}$ the calculated photon spectra are compared to the MSL-RAD measurements. Again, GEANT4, HZETRN/ OLTARIS, and PHITS data are compatible. The photon results from HZETRN/OLTARIS appear to deviate from the MonteCarlo results above $1 \mathrm{GeV}$ by about one order of magnitude and below $10 \mathrm{MeV}$. The discrepancies at energies above $1 \mathrm{GeV}$ are associated mainly with differing neutral pion production models, where the HZETRN/OLTARIS model needs improvement. The HZETRN/OLTARIS model also does not currently include photon production from nuclear collisions, explaining the underestimate below $10 \mathrm{MeV}$.

\subsection{Charged particle spectra}

Figures 5-10 show the calculated charged particle spectra and the values measured by the RAD instrument (Ehresmann et al. 2014). The particle fluxes in Figures 5-9 from PHITS,
HZETRN/OLTARIS, and GEANT4 are averaged over the acceptance angle for the determination of the charged particle fluxes with RAD, i.e. zenith angles below $30^{\circ}$. OLTARIS2013 data is averaged over the upper hemisphere, i.e. zenith angles below $90^{\circ}$. Particle fluxes in Figure 10 are averaged over all incident angles. The averaged particle fluxes over all incident angles can be significantly different, especially at high energies where most or even all particles are incident at small zenith angles and there are very few or no albedo particles.

Figures 5 and 6 contain the spectra of: protons, deuterons, tritons, ${ }^{3} \mathrm{He}$, and ${ }^{4} \mathrm{He}$-particles. The energy range is limited to $E<110 \mathrm{MeV} / \mathrm{n}$ to facilitate a comparison to the RAD data. In Figure 7 the same data is illustrated for a much wider energy range up to $20 \mathrm{GeV} / \mathrm{n}$. The GEANT4 results were obtained using the Intranuclear Cascade Model of Liège (INCL; Boudard et al. 2002; Leray et al. 2013; Mancusi et al. 2014) (setup [1] from Table 2) as it was found in this work that using other nuclear interaction models available in GEANT4 did not result in secondary hydrogen and helium isotope production comparable to the RAD measurements. In general, good agreement between GEANT4 predictions and RAD data is found for 
D. Matthiä et al.: The Martian Surface Radiation Environment - Models and Measurements

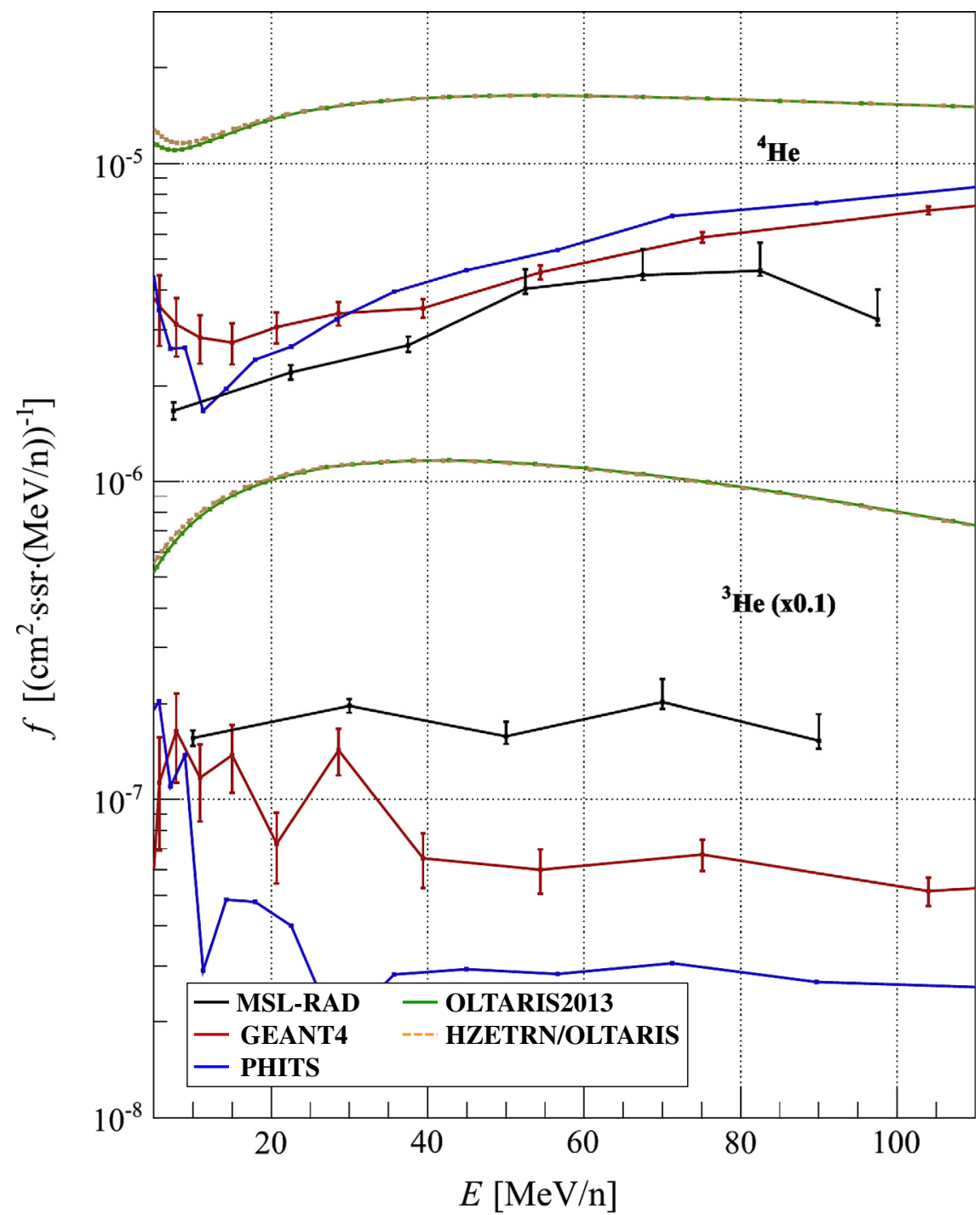

Fig. 6. ${ }^{4} \mathrm{He}$ and ${ }^{3} \mathrm{He}$ (scaled by 0.1 ) spectra for zenith angles smaller than $30^{\circ}$ on the Martian surface measured between 19 August 2012 and 17 February 2013 by MSL-RAD (black, Ehresmann et al. 2014) and calculated for the same period by different simulation tools: GEANT4 (red), PHITS (blue), OLTARIS2013 (green), HZETRN/OLTARIS (ocher). The data have been scaled by the factors given in parentheses.

the lighter nuclei shown in Figures 5 and 6, with the exception of ${ }^{3} \mathrm{He}$ for which the measured flux seems to be underestimated by almost one order of magnitude by GEANT4 at energies above $20 \mathrm{MeV} / \mathrm{n}$. PHITS reproduces the measured deuteron flux nicely but shows large discrepancies in case of the other nuclei. Especially for triton and ${ }^{3} \mathrm{He}$ the calculated values are more than one order of magnitude lower than the measurements.

Although all codes appear to overestimate the measured proton results, the results of HZETRN/OLTARIS are the largest of the model results. This is caused in part by differing nuclear models as well as by the lack of 3D effects in this version of HZETRN. Future work could use the 3DHZETRN code (Wilson et al. 2014, 2015) to gauge the impact of 3D corrections for these atmospheric/surface scenarios. The tendency of all models to overestimate the measured proton spectra, especially at these energies, might also be attributable to uncertainties in the primary GCR spectrum and atmospheric composition and thickness.

For deuterons, all codes appear to have better agreement with the measured results. However, the HZETRN/OLTARIS results are again larger than all other results, pointing to differing nuclear models and 3D effects not accounted for in this set of calculations.

The largest model errors (more than a factor of 10) occur for tritons and ${ }^{3} \mathrm{He}$ which are not present in the modeled primary GCR. Therefore, these model spectra are purely secondary reaction products. The HZETRN/OLTARIS results again provide the largest values; the overestimate is associated mainly with a cluster-knockout model (Cucinotta et al. 1996) that appears to need further study and improvement. On the other hand, PHITS results appear to underestimate the measurement by about the same amount as HZETRN/OLTARIS overestimates. Geant 4 is also below the measured values.

Comparing the results for the light nuclei at high energies (Fig. 7) it is obvious that there is a good agreement between the different codes only for particles which are dominated by the primary components: protons and alpha particles. At high energies the flux of these particles is very similar to the flux of primaries on top of the Martian atmosphere. For deuterons, tritons, and ${ }^{3} \mathrm{He}$, on the other hand, the differences between the models can reach almost an order of magnitude. As the $\mathrm{RAD}$ instrument cannot provide data at these energies it is not possible to decide which of the predictions is the more 


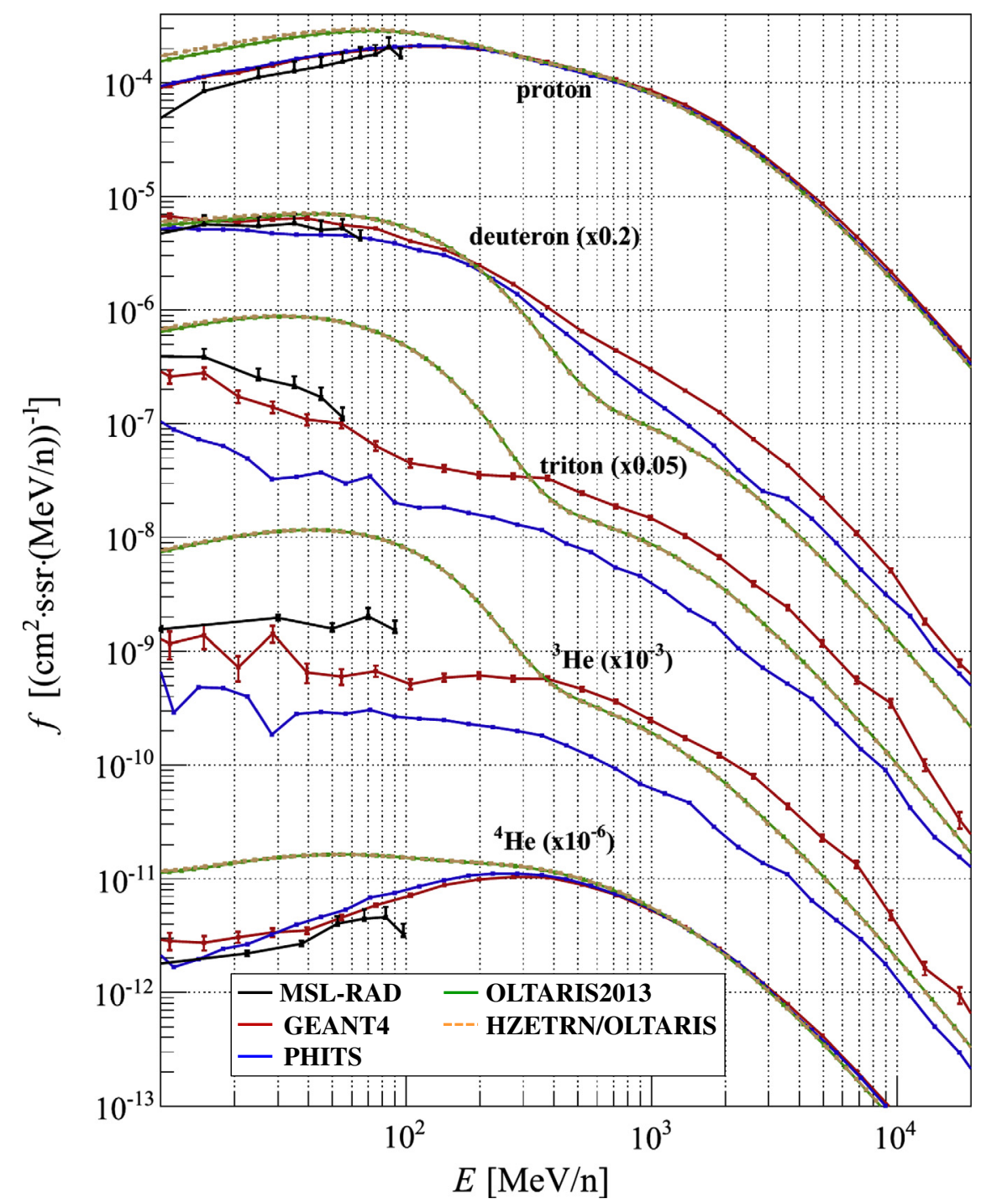

Fig. 7. Same data as Figures 5 and 6 but for an energy range from $10 \mathrm{MeV} / \mathrm{n}$ to $20 \mathrm{GeV} / \mathrm{n}$.

accurate. However, the contribution of these isotopes to the total dose is small and the effect of the differences in the particle flux on the total radiation exposure is negligible as will be shown below.

For the fluxes of heavier nuclei on the surface of Mars (Figs. 8 and 9) we observe a reasonable agreement between GEANT4, PHITS, OLTARIS, HZETRN/OLTARIS and the MSL-RAD data for nuclei up to aluminum $(Z=13)$ upper three panels in Figure 8. For the group of silicon to chromium $(Z=14-24)$ GEANT4 reveals good agreement with the measurement: OLTARIS2013 and HZETRN/OLTARIS seem to be compatible and PHITS overestimates the measured data. For nuclei with $Z>24$ all codes seem to underestimate the experimental data. However, the low number of high- $Z$ particles leads to a comparatively large statistical uncertainty in the measurement. At higher energies (Fig. 9) GEANT4 data is significantly higher than the results of the other models for the groups of ( $\mathrm{Li}, \mathrm{Be}, \mathrm{B}), Z=9-13$ and $Z>24$.

Figure 10 shows the calculated particle spectra for electrons/positrons $\left(e^{-}, e^{+}\right)$, muons $\left(\mu^{-}, \mu^{+}\right)$, and pions $\left(\pi^{-}, \pi^{+}\right)$. For these particles no measurement is currently available. The comparison of the models providing information about these particles (GEANT4, PHITS, and HZETRN/OLTARIS) shows good agreement for electrons and positrons over almost the whole energy range. For muons, however, agreement is only observed at energies above $1 \mathrm{GeV}$ and for pions at energies above $3 \mathrm{GeV}$. At lower energies the calculated particle spectra differ by several orders of magnitude.

\subsection{Dose rate}

Figure 11 and Table 2 summarize the results of the calculation of dose rates on the Martian surface and the comparison to the RAD measurements. In Figure 11, the contribution of different particle species to the total dose rates calculated with the different transport models is illustrated. The results from GEANT4 shown in Figure 11 were obtained with the physics list setup [1] for which the best agreement with the measured particle flux was found. For OLTARIS2013 for which only downward fluxes were available, it was assumed that these represent the full radiation field.

The values derived from the different transport models for the total dose equivalent rates $(0.51-0.60 \mathrm{mSv} / \mathrm{d})$ as well as the dose rate in tissue $(0.16-0.20 \mathrm{mGy} / \mathrm{d})$ and the dose rate in silicon $(0.13-0.16 \mathrm{mGy} / \mathrm{d})$ agree within $20 \%$. The contributions of the different particle types to the total dose, however, are 
D. Matthiä et al.: The Martian Surface Radiation Environment - Models and Measurements

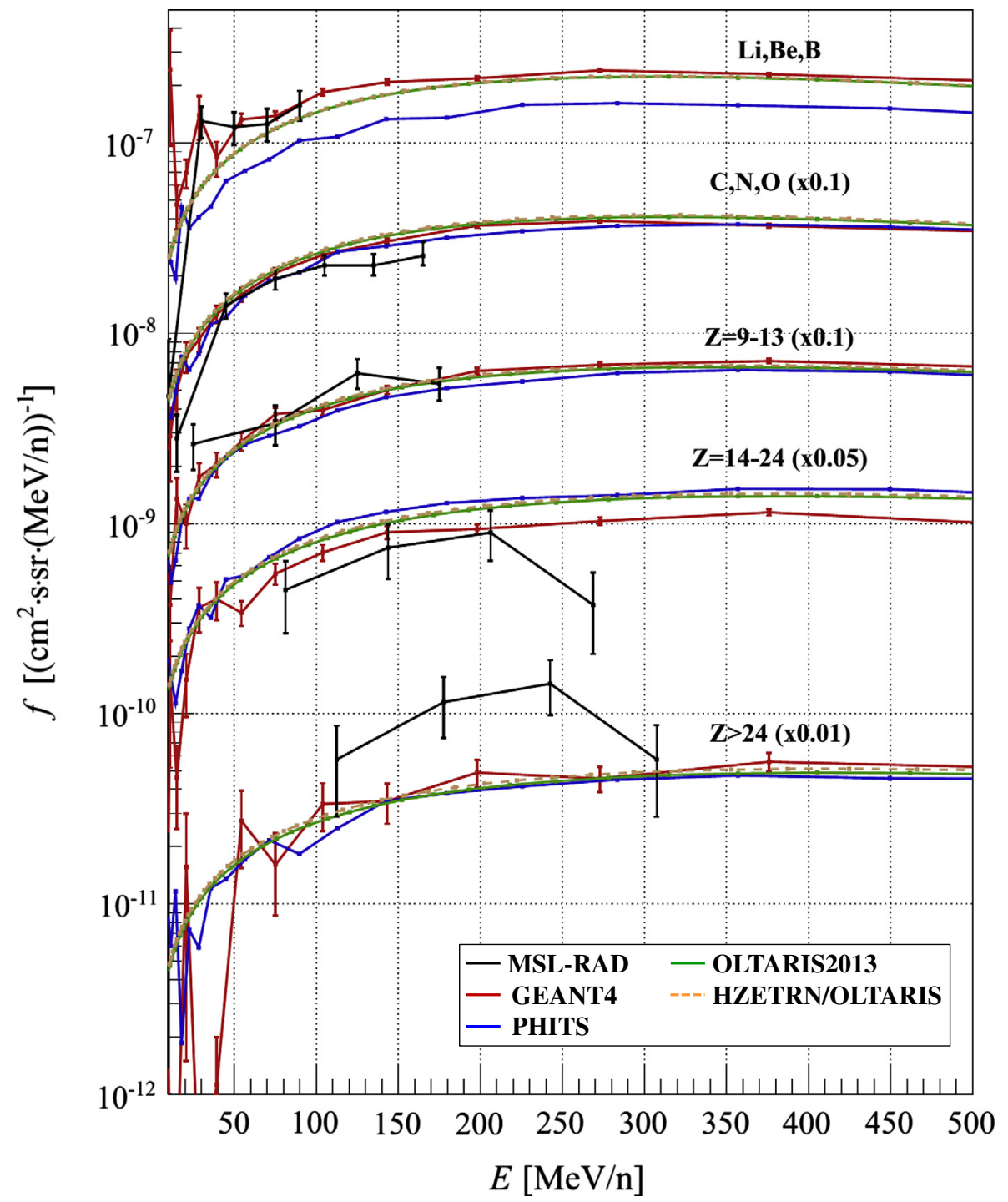

Fig. 8. Particle spectra for zenith angles smaller than $30^{\circ}$ on the Martian surface measured between 19 August 2012 and 17 February 2013 by MSL-RAD (black, Ehresmann et al. 2014) and calculated for the same period by different simulation tools: GEANT4 (red), PHITS (blue), OLTARIS2013 (green), HZETRN/OLTARIS (ocher). Different nuclei are grouped together and scaled by the factors given in parentheses.

in some cases remarkably different. Particularly the contribution from heavy ions to the dose equivalent rate derived from the OLTARIS2013 results differs from the HZETRN/ OLTARIS, PHITS, and GEANT4 results by a factor of two to four which is a consequence of the simplified geometry used to generate the OLTARIS2013 results. On the other hand, the contribution of neutrons from OLTARIS2013 is only about $10 \%-15 \%$ of the HZETRN/OLTARIS, PHITS, and GEANT4 results. Both of these observations are a consequence of the simplified geometry used to generate the OLTARIS2013 results. The dose equivalent rates from HZETRN/OLTARIS, PHITS, and GEANT4 tend to agree better but also show some differences in the contribution of neutrons, which is about $20 \%$ $(0.05 \mathrm{mSv} / \mathrm{d})$ lower for GEANT4 and HZETRN/OLTARIS, and electrons, which is about $50 \%(0.02 \mathrm{mSv} / \mathrm{d})$ lower for HZETRN/OLTARIS compared to PHITS and GEANT4. The quality factor for the whole radiation field derived from the different numerical models lies between $Q=2.7$ (GEANT4) and $Q=3.2$ (PHITS and HZETRN/OLTARIS). As the quality factor in the RAD measurement is derived from a limited acceptance angle we have also calculated the contribution to the dose rate and the dose equivalent rate from particles with an incident zenith angle below $30^{\circ}$. These values were calculated with all models and are given in Table 2 in brackets. The results of GEANT4 suggest that the quality factor for the limited sensitive solid angle is higher $(Q=3.0-3.5)$ than that for the whole radiation field $(Q=2.7-3.0)$. Dose rates calculated from PHITS $(Q=3.0$ and $Q=3.4)$ and HZETRN/OLTARIS also show this feature, although for HZETRN/OLTARIS with lower significance: $Q=3.2$ for the limited solid angle and $Q=3.0$ for the whole field. A higher quality factor for the limited zenith angle range would imply that the RAD derived quality factor overestimates the quality factor of the whole radiation field by about $10-25 \%$. If we consider the fact that the particles with the highest quality factor are heavy ions, which are primarily downward directed either as fragments of GCR projectiles or as fragments of atmospheric target nuclei, a lower quality factor for the whole field compared to the restricted solid angle is plausible. Neutrons which have a high quality factor as well, on the other hand, are almost isotropically distributed. Thus, if the contribution of neutrons to the total dose is higher in some models, the effect of the restricted sensitive angle on the calculated quality factor is lower. This is the case for the GEANT4 setup [5] which uses the Bertini cascade 


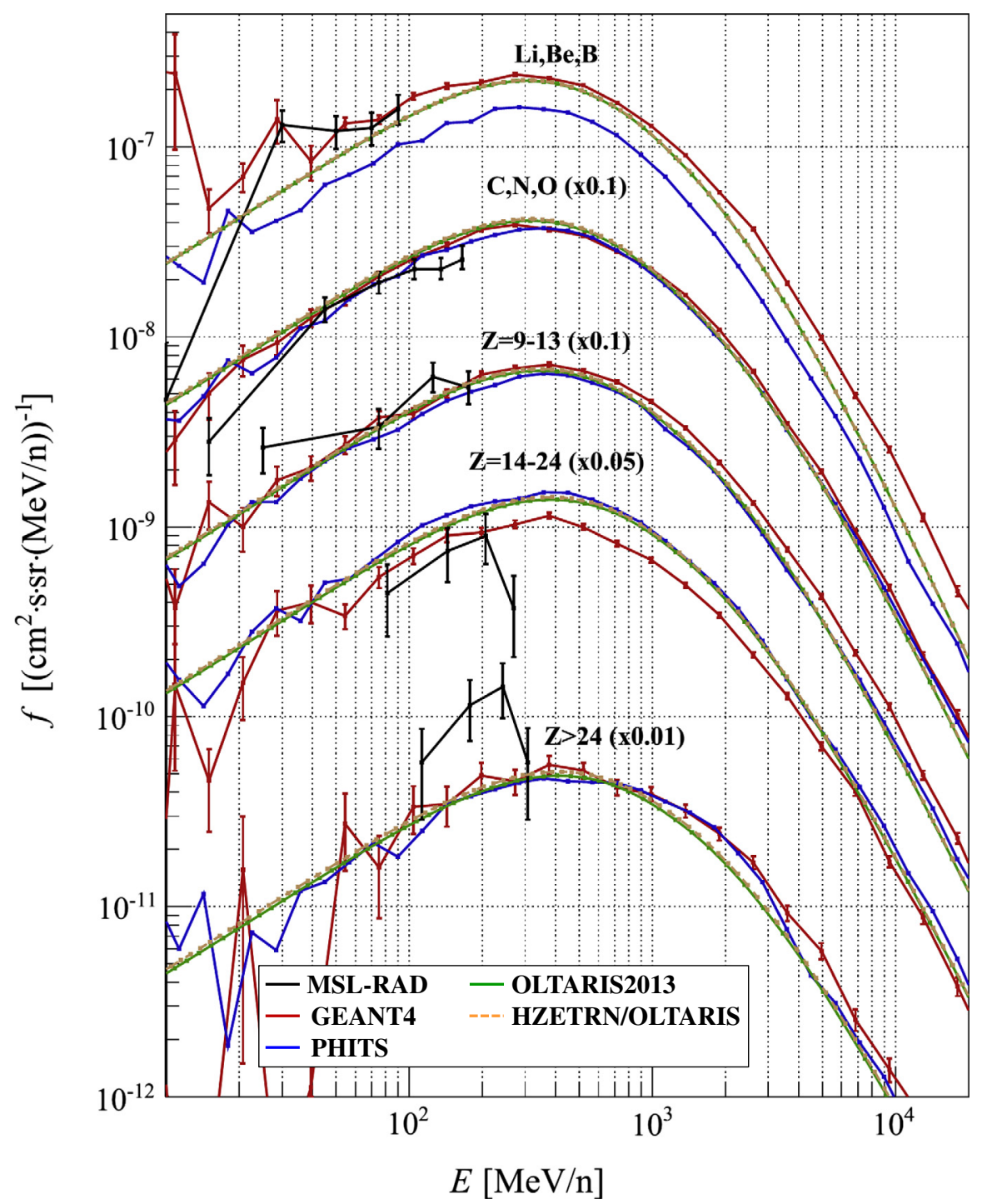

Fig. 9. Same as Figure 8 but for an energy range from $10 \mathrm{MeV} / \mathrm{n}$ to $20 \mathrm{GeV} / \mathrm{n}$.

model and for which the contribution of neutrons to the total dose is much higher than for the other GEANT4 setups $(0.27 \mathrm{mSv} / \mathrm{d}$ compared to $\sim 0.18 \mathrm{mSv} / \mathrm{d})$. Consequentially the difference in the quality factor from the limited zenith angle range $(Q=3.2)$ and the whole field is decreased $(Q=3.0)$. All numerical results for the quality factor are compatible with the experimental values within the uncertainty of the RAD measurements.

Simonsen et al. (1990) have estimated the skin dose equivalent during solar minimum (GCR maximum) conditions on the Martian surface for a shielding of $22 \mathrm{~g} / \mathrm{cm}^{2} \mathrm{CO}_{2}$ to be $11.3 \mathrm{rem} /$ year $(0.31 \mathrm{mSv} / \mathrm{d})$ which is significantly lower than the values measured by RAD and estimated in this work. Even more so if we consider the fact that the measurements and the calculations of this work have not been performed for solar minimum for which higher dose rates are expected. Townsend et al. (2011) estimated the skin dose rate on Mars during solar minimum for an atmospheric depth of $20 \mathrm{~g} / \mathrm{cm}^{2}$ to be $0.305 \mathrm{mGy} / \mathrm{d}$ and the effective dose rate to be $0.726 \mathrm{mSv} / \mathrm{d}$. While skin dose rate estimated by Townsend et al. (2011) is about $50 \%$ greater than the values measured by RAD and calculated in this work, the effective dose rate is only about $15 \%$ greater than the measurement and about $40 \%$ greater than the calculations in this work. Parts of these discrepancies could be explained by differences in the solar modulation of the GCR. Also, Simonsen et al. (1990) and Townsend et al. (2011) have used different models for the primary GCR spectra, CREME (Adams et al. 1981) and Badhwar-O'Neil 2006 (O’Neill 2006), and the transport, BRYNTRN (Wilson et al. 1989) and an older version of HZTREN (Nealy et al. 2007). Unfortunately, in retrospect it is impossible to disentangle the influences of the different factors from the results and what could have led to the observed discrepancies. In case of Townsend et al. (2011) a direct comparison to the results of this work is additionally complicated by the fact that they calculated the dose rate for vertically incident particles only and that the calculated organ dose values are affected by the selfshielding of the applied water phantom. Future work will show how the use of a numerical phantom or organ dose conversion factors influences the resulting dose rates for the Martian surface.

\section{Summary}

We present the first comprehensive comparison of results of different numerical radiation transport models, GEANT4, PHITS, OLTARIS2013, HZETRN/OLTARIS of particle 
D. Matthiä et al.: The Martian Surface Radiation Environment - Models and Measurements
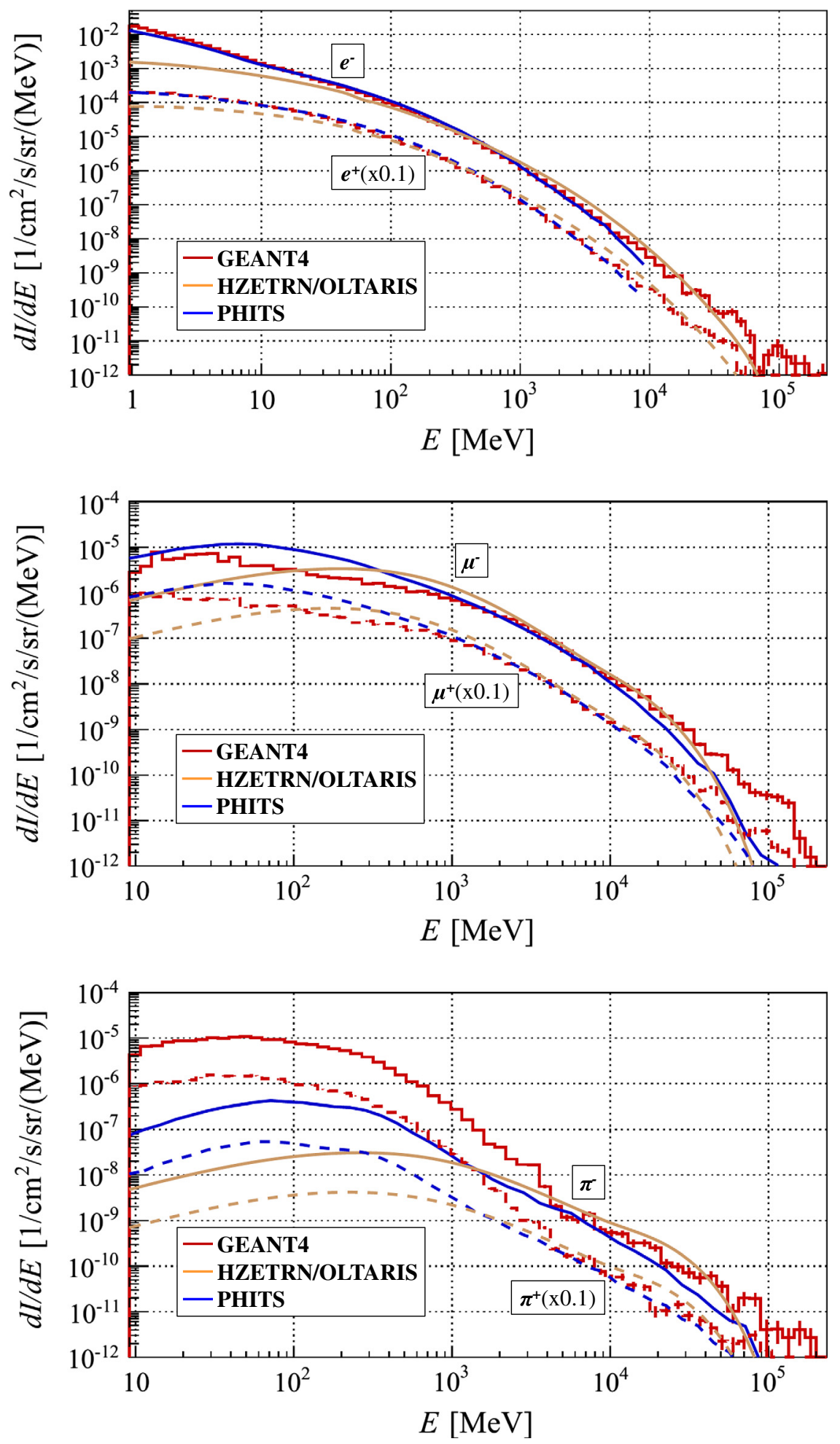

Fig. 10. Particle spectra on the Martian surface calculated for the time period between 19 August 2012 and 17 February 2013 by different simulation tools: GEANT4 (red), PHITS (blue), HZETRN/OLTARIS (ocher). Particle types are, from top to bottom: electron/positron, $\mu^{-} / \mu^{+}$, $\pi^{-} / \pi^{+}$. Positron, $\mu^{+}$and $\pi^{+}$are indicated by the dashed lines and scaled by 0.1 for separation.

spectra over a wide energy range and dose rates caused by galactic cosmic radiation on the surface of Mars with direct measurements on the surface of Mars from the MSL-RAD instrument. Although good agreement is found in many cases for GEANT4, PHITS, and HZETRN/OLTARIS, some models still show large, sometimes order of magnitude, discrepancies in certain particle spectra. We have also found that comparison with these RAD data is helping us make better choices of input parameters and physical models, and thus improving agreement of the simulation results with the data. The first-ever comparison between experimental data from the surface of Mars and model calculations is helping us to improve the reliability of numerical model predictions for particle spectra and radiation exposure on Mars as well as for other space travel scenarios. 
(a)
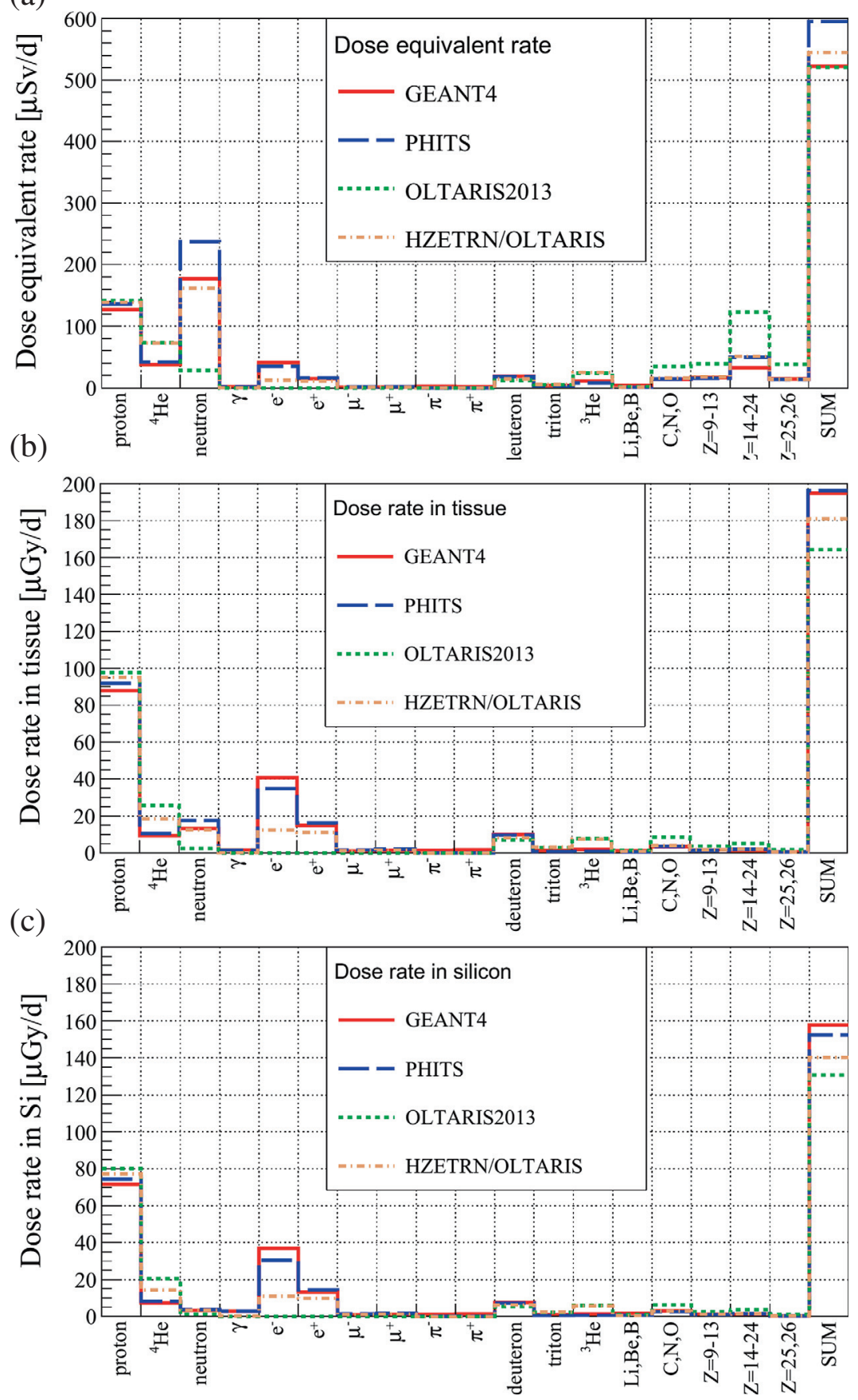

Fig. 11. Contribution of different particle types to the dose equivalent rate in tissue (a, in $\mu \mathrm{Sv} / \mathrm{d}$ ), dose rates (in $\mu \mathrm{Gy} / \mathrm{d}$ ) in tissue (b), and silicon (c) calculated with different transport models for the Martian surface (atmospheric shielding: $22 \mathrm{~g} / \mathrm{cm}^{2}$ ). MSL-RAD measured a dose rate in tissue of $0.21 \pm 0.04 \mathrm{mGy} /$ day and a dose equivalent rate of $0.64 \pm 0.12 \mathrm{mSv} /$ day Hassler et al. 2014).

It was found that the selection of different physical model lists in GEANT4 in most cases does not affect the resulting radiation exposure severely: a maximum difference of $20 \%$ is calculated in the dose equivalent rate for the application of the Bertini cascade model; the dose rate in tissue agrees within approximately $5 \%$ for all GEANT4 model lists. In some cases, however, the model selection in GEANT4 affects the resulting prediction of secondary particle spectra on the surface: the application of the Intranuclear Cascade Model of Liège (INCL) results in a strongly improved agreement of hydrogen and helium isotope spectra with the RAD measurements; using the Bertini cascade model for inelastic interactions of neutrons and protons at energies below $10 \mathrm{GeV}$ leads to strongly enhanced neutron fluxes on the surface and, as a result, to an increased quality factor. Based on the current results of RAD, however, it cannot be decided which of the models is most accurate concerning the prediction of neutron fluxes.

No significant difference in the prediction of particle spectra and dose rate was found for the two models of galactic cosmic radiation. However, from other published results it can be assumed that this is special for the solar modulation conditions for which the study was performed and it can be assumed that this could be different, e.g. for solar minimum conditions. 
Despite the agreement between calculated and measured dose it is necessary to further improve the numerical models and to continue the validation against experimental data. Measurements, especially for neutrons below $10 \mathrm{MeV}$ and heavy ions in the range above several hundreds of $\mathrm{MeV} / \mathrm{n}$, would help to develop the models at these energies where in some cases large discrepancies were observed in this work. This energy range is also expected to become more important in case of heavier shielded environments, e.g. underground or in radiation shelters. Additionally, as stated above, the concept of dose and quality factor for the description of biological damage and radiation risk might not be fully adequate for space radiation applications. The contribution of particles and the importance of energy ranges might alter in different radiation risk models and experimental data would help to estimate the accuracy of the numerical models.

Acknowledgements. RAD is supported by NASA (HEOMD) under JPL Subcontract 1273039 to Southwest Research Institute and in Germany by DLR and DLR's Space Administration Grants 50QM0501 and 50QM1201 to the Christian Albrechts University, Kiel. Part of this research was carried out at the Jet Propulsion Laboratory, California Institute of Technology, under a contract with the National Aeronautics and Space Administration.

The authors would like to thank the Sodankyla Geophysical Observatory and the website team (http://cosmicrays.oulu.fi) for providing the Oulu neutron monitor data.

The editor thanks Guillaume Gronoff and an anonymous referee for their assistance in evaluating this paper.

\section{References}

Adamczyk, A.M., R.B. Norman, S.I. Sriprisan, L.W. Townsend, J.W. Norbury, S.R. Blattnig, and T.C. Slaba. NUCFRG3: light ion improvements to the nuclear fragmentation model. Nucl. Instrum. Methods Phys. Res. Sect. A, 678, 21-32, 2012, DOI: $10.1016 /$ j.nima.2012.02.021.

Adams Jr., J.H., R. Silberberg, and C.H. Tsao. Cosmic ray effects on microelectronics. Part 1: the near-Earth particle environment. NASA STI/Recon Technical Report N, 81, 34134, 1981.

Agostinelli, S., J. Allison, and K. Amako. Geant4-a simulation toolkit. Nucl. Instrum. Methods Phys. Res. Sect. A, 506, 250-303, 2003, DOI: 10.1016/S0168-9002(03)01368-8.

Allison, J., K. Amako, J. Apostolakis, H. Araujo, P.A. Dubois, et al. Geant4 developments and applications. IEEE Trans. Nucl. Sci., 53, 270-278, 2006, DOI: 10.1109/TNS.2006.869826.

Boudard, A., J. Cugnon, S. Leray, and C. Volant. Intranuclear cascade model for a comprehensive description of spallation reaction data. Phys. Rev. C, 66 (4), 44615, 2002,

DOI: 10.1103/PhysRevC.66.044615.

Boudard, A., J. Cugnon, J.C. David, S. Leray, and D. Mancusi. New potentialities of the Liège intranuclear cascade model for reactions induced by nucleons and light charged particles. Phys. Rev. C, 87 (1), 014606, 2013,

DOI: 10.1103/PhysRevC.87.014606.

Bougher, S.W., R.G. Roble, E.C. Ridley, and R.E. Dickinson. The Mars thermosphere: 2 general circulation with coupled dynamics and composition. J. Geophys. Res. [Solid Earth], 95 (B9), 14811-14827, 1990, DOI: 10.1029/JB095iB09p14811.

Cucinotta, F.A. Calculation of Cosmic-Ray Helium Transport in Shielding Materials. Report NASA-TP-3354, NASA, 1993.

Cucinotta, F.A., L.W. Townsend, J.W. Wilson, J.L. Shinn, G.D. Badhwar, and R.R. Dubey. Light ion components of the galactic cosmic rays: nuclear interactions and transport theory. Adv. Space Res., 17 (2), 77-86, 1996, DOI: $10.1016 / 0273-1177(95) 00515-G$

Cucinotta, F.A., S. Hu, N.A. Schwadron, K. Kozarev, L.W. Townsend, and M.H.Y. Kim. Space radiation risk limits and
Earth-Moon-Mars environmental models. Space Weather, 8, 1-121, 2010, DOI: 10.1029/2010SW000572.

Cucinotta, F.A., L.J. Chappell, and M.J. Kim. Space Radiation Cancer Risk Projections and Uncertainties - 2010. Report NASA TP 2011-216155, NASA, 2011.

Dartnell, L.R., L. Desorgher, J.M. Ward, and A.J. Coates. Martian sub-surface ionising radiation: biosignatures and geology. Biogeosciences, 4 (4), 545-558, 2007, DOI: 10.5194/bg-4-545-2007.

Durante, M., and F.A. Cucinotta. Heavy ion carcinogenesis and human space exploration. Nat. Rev. Cancer, 8, 465-472, 2008, DOI: $10.1038 / \mathrm{nrc} 2391$.

Ehresmann, B. The Martian radiation environment - early mars and future measurements with the radiation assessment detector. Thesis, Christian-Albrechts-Universität zu Kiel, 2012.

Ehresmann, B., C. Zeitlin, D.M. Hassler, R.F. Wimmer-Schweingruber, E. Böhm, et al. Charged particle spectra obtained with the Mars Science Laboratory Radiation Assessment Detector (MSL/RAD) on the surface of Mars. J. Geophys. Res. [Planets], 119 (3), 468-479, 2014, DOI: 10.1002/2013JE004547.

Gronoff, G., R.B. Norman, and C.J. Mertens. Computation of cosmic ray ionization and dose at Mars. I: A comparison of HZETRN and Planetocosmics for proton and alpha particles. Adv. Space Res., 55 (7), 1799-1805, 2015,

DOI: 10.1016/j.asr.2015.01.028.

Grotzinger, J.P., J. Crisp, A.R. Vasavada, R.C. Anderson, C.J. Baker, et al. Mars Science Laboratory mission and science investigation. Space Sci. Rev., 170 (1-4), 5-56, 2012,

DOI: $10.1007 / \mathrm{s} 11214-012-9892-2$.

Guo, J., C. Zeitlin, R.F. Wimmer-Schweingruber, D.M. Hassler, A. Posner, et al. Variations of dose rate observed by MSL/RAD in transit to Mars. $A \& A, \mathbf{5 7 7}, \mathrm{A} 58,2015$,

DOI: $10.1051 / 0004-6361 / 201525680$.

Gómez-Elvira, J., C. Armiens, I. Carrasco, M. Genzer, F. Gómez, et al. Curiosity's rover environmental monitoring station: overview of the first 100 sols. J. Geophys. Res. [Planets], 119 (7), 2013JE004576, 2014, DOI: 10.1002/2013JE004576.

Haberle, R.M., J.B. Pollack, J.R. Barnes, R.W. Zurek, C.B. Leovy, J.R. Murphy, H. Lee, and J. Schaeffer. Mars atmospheric dynamics as simulated by the NASA Ames General Circulation Model: 1. the zonal mean circulation. J. Geophys. Res. [Planets], 98 (E2), 3093-3123, 1993, DOI: 10.1029/92JE02946.

Haberle, R.M., J. Gomez-Elvira, M.D. Juarez, A.M. Harri, J.L. Hollingsworth, et al. Preliminary interpretation of the REMS pressure data from the first 100 sols of the MSL mission. J. Geophys. Res. [Planets], 119 (3), 440-453, 2014, DOI: $10.1002 / 2013 \mathrm{je} 004488$.

Hassler, D.M., C. Zeitlin, R.F. Wimmer-Schweingruber, S. Bottcher, C. Martin, et al. The Radiation Assessment Detector (RAD) investigation. Space Sci. Rev., 170 (1-4), 503-558, 2012, DOI: $10.1007 / \mathrm{s} 11214-012-9913-1$.

Hassler, D.M., C. Zeitlin, R.F. Wimmer-Schweingruber, B. Ehresmann, S. Rafkin, et al. Mars' surface radiation environment measured with the Mars Science Laboratory's Curiosity rover. Science, 343 (6169), 2014, DOI: $10.1126 /$ Science. 1244797.

ICRP. 1990 Recommendations of the International Commission on Radiological Protection. Annals of the ICRP, 21 (1-3), 1-201, 1991.

ICRP. The 2007 Recommendations of the International Commission on Radiological Protection. Annals of the ICRP, 37 (2-4), 1-332, 2007.

Justus, C.G., and D.L. Johnson. Mars Global Reference Atmospheric Model 2001 Version (Mars-GRAM 2001): users guide, NASA/TM-2001-210961, 2001.

Justus, C.G., A. Duvall, and V.W. Keller. Validation of Mars Global Reference Atmospheric Model (Mars-GRAM 2001) and planned new features. Adv. Space Res., 38, 2633-2638, 2006, DOI: 10.1016/j.asr.2006.07.007.

Kim, M.H.Y., F.A. Cucinotta, H.N. Nounu, C. Zeitlin, D.M. Hassler, et al. Comparison of Martian surface ionizing radiation measurements from MSL-RAD with Badhwar-O'Neill 
2011/HZETRN model calculations. J. Geophys. Res. [Planets], 119 (6), 1311-1321, 2014, DOI: 10.1002/2013je004549.

Köhler, J., C. Zeitlin, B. Ehresmann, R.F. Wimmer-Schweingruber, D.M. Hassler, et al. Measurements of the neutron spectrum on the Martian surface with MSL/RAD. J. Geophys. Res. [Planets], 119 (3), 594-603, 2014, DOI: 10.1002/2013JE004539.

Köhler, J., B. Ehresmann, C. Zeitlin, R.F. Wimmer-Schweingruber, D.M. Hassler, et al. Measurements of the neutron spectrum in transit to Mars on the Mars Science Laboratory. Life Sciences in Space Research, 5, 6-12, 2015, DOI: 10.1016/j.1ssr.2015.03.001.

Leray, S., D. Mancusi, P. Kaitaniemi, J.C. David, A. Boudard, B. Braunn, and J. Cugnon. Extension of the Liège Intra Nuclear Cascade model to light ion-induced collisions for medical and space applications. J. Phys: Conf. Ser., 420 (1), 12065, 2013, DOI: $10.1088 / 1742-6596 / 420 / 1 / 012065$.

Mancusi, D., A. Boudard, J. Cugnon, J.-C. David, P. Kaitaniemi, and S. Leray. Extension of the Liège intranuclear-cascade model to reactions induced by light nuclei. Phys. Rev. C, 90 (5), 54602, 2014, DOI: 10.1103/PhysRevC.90.054602.

Matthiä, D., T. Berger, A.I. Mrigakshi, and G. Reitz. A ready-to-use galactic cosmic ray model. Adv. Space Res., 51, 329-338, 2013, DOI: $10.1016 /$ j.asr.2012.09.022.

McKenna-Lawlor, S., P. Goncalves, A. Keating, B. Morgado, D. Heynderickx, et al. Characterization of the particle radiation environment at three potential landing sites on Mars using ESA's MEREM models. Icarus, 218 (1), 723-734, 2012a, DOI: $10.1016 /$ j.icarus.2011.04.004

McKenna-Lawlor, S., P. Gonçalves, A. Keating, G. Reitz, and D. Matthiä. Overview of energetic particle hazards during prospective manned missions to Mars. Planet. Space Sci., 63-64, 123-132, 2012b, DOI: 10.1016/j.pss.2011.06.017.

Mrigakshi, A.I., D. Matthiä, T. Berger, G. Reitz, and R.F. WimmerSchweingruber. Assessment of galactic cosmic ray models. J. Geophys. Res. [Space Phys.], 117 (A8), A08109, 2012, DOI: 10.1029/2012JA017611.

Mrigakshi, A.I., D. Matthiä, T. Berger, G. Reitz, and R.F. WimmerSchweingruber. Estimation of galactic cosmic ray exposure inside and outside the Earth's magnetosphere during the recent solar minimum between solar cycles 23 and 24. Adv. Space Res., 52, 979-987, 2013a, DOI: 10.1016/j.asr.2013.05.007.

Mrigakshi, A.I., D. Matthiä, T. Berger, G. Reitz, and R.F. WimmerSchweingruber. How galactic cosmic ray models affect the estimation of radiation exposure in space. Adv. Space Res., 51, 825-834, 2013b, DOI: 10.1016/j.asr.2012.10.017.

Nara, Y., N. Otuka, A. Ohnishi, K. Niita, and S. Chiba. Relativistic nuclear collisions at $10 \mathrm{~A} \mathrm{GeV}$ energies from $\mathrm{p}+\mathrm{Be}$ to $\mathrm{Au}+\mathrm{Au}$ with the hadronic cascade model. Phys. Rev. C, 61 (2), 024901, 1999, DOI: 10.1103/PhysRevC.61.024901.

National Research Council. S. R. S. P. Radiobiological factors in manned space flight. Report. In: W.H. Langham, Editor. Publication (National Research Council (U.S.)); no. 1487, National Academy of Sciences, National Research Council, Washington, 1967.

Nealy, J., F. Cucinotta, J. Wilson, F. Badavi, T. Dachev, B. Tomov, S. Walker, G.D. Angelis, S. Blattnig, and W. Atwell. Preengineering spaceflight validation of environmental models and the 2005 HZETRN simulation code. Adv. Space Res., 40 (11), 1593-1610, 2007, DOI: 10.1016/j.asr.2006.12.029.

Niita, K., S. Chiba, T. Maruyama, T. Maruyama, H. Takada, T. Fukahori, Y. Nakahara, and A. Iwamoto. Analysis of the $(\mathrm{N}, \mathrm{xN}$ ') reactions by quantum molecular dynamics plus statistical decay model. Phys. Rev. C, 52 (5), 2620-2635, 1995, DOI: 10.1103/PhysRevC.52.2620.

Niita, K., T. Sato, H. Iwase, H. Nose, H. Nakashima, and L. Sihver. PHITS - a particle and heavy ion transport code system. Radiat. Meas., 41, 1080-1090, 2006,

DOI: $10.1016 /$ j.radmeas.2006.07.013

Norman, R.B., T.C. Slaba, and S.R. Blattnig. An extension of HZETRN for cosmic ray initiated electromagnetic cascades. $A d v$. Space Res., 51, 2251-2260, 2013, DOI: 10.1016/j.asr.2013.01.021.
Ogawa, T., T. Sato, S. Hashimoto, and K. Niita. Development of a reaction ejectile sampling algorithm to recover kinematic correlations from inclusive cross-section data in Monte-Carlo particle transport simulations. Nucl. Instrum. Methods Phys. Res. Sect. A, 763, 575-590, 2014, DOI: 10.1016/j.nima.2014.06.088.

O'Neill, P.M. Badhwar O'Neill galactic cosmic ray model update based on advanced composition explorer (ACE) energy spectra from 1997 to present. Adv. Space Res., 37, 1727-1733, 2006, DOI: $10.1016 /$ j.asr.2005.02.001

O’Neill, P.M. Badhwar-O'Neill 2010 galactic cosmic ray flux model revised. IEEE Trans. Nucl. Sci., 57 (6), 3148-3153, 2010, DOI: $10.1109 /$ TNS.2010.2083688.

Posner, A., D. Hassler, D. McComas, S. Rafkin, R. WimmerSchweingruber, E. Böhm, S. Böttcher, S. Burmeister, W. Dröge, and B. Heber. A high energy telescope for the Solar Orbiter. Adv. Space Res., 36 (8), 1426-1431, 2005, Solar Encounter, Solar-B and \{STEREO\}, DOI: 10.1016/j.asr.2004.11.040.

Rafkin, S.C.R., C. Zeitlin, B. Ehresmann, D. Hassler, J.N. Guo, et al. Diurnal variations of energetic particle radiation at the surface of Mars as observed by the Mars Science Laboratory Radiation Assessment Detector. J. Geophys. Res. [Planets], 119 (6), 1345-1358, 2014, DOI: 10.1002/2013je004525.

Sato, T., K. Niita, N. Matsuda, S. Hashimoto, Y. Iwamoto, et al. Particle and heavy ion transport code system, PHITS, version 2.52. J. Nucl. Sci. Technol. , 50, 913-923, 2013, DOI: $10.1080 / 00223131.2013 .814553$.

Sato, T., R. Kataoka, H. Yasuda, S. Yashiro, T. Kuwabara, D. Shiota, and Y. Kubo. Air shower simulation for WASAVIES: warning system for aviation exposure to solar energetic particles. Radiat. Prot. Dosim., 161 (1-4), 274-278, 2014, DOI: $10.1093 / \mathrm{rpd} / \mathrm{nct} 332$.

Shibata, K., O. Iwamoto, T. Nakagawa, N. Iwamoto, A. Ichihara, et al. JENDL-4.0: a new library for nuclear science and engineering. J. Nucl. Sci. Technol., 48 (1), 1-30, 2011, DOI: $10.1080 / 18811248.2011 .9711675$.

Simonsen, L.C., J.E. Nealy, L.W. Townsend, and J.W. Wilson. Space radiation-dose estimates on the surface of Mars. Journal of Spacecraft and Rockets, 27 (4), 353-354, 1990, DOI: $10.2514 / 3.26149$.

Simpson, J.A. Elemental and isotopic composition of the galactic cosmic rays. Annual Review of Nuclear and Particle Science, 33 323-382, 1983, DOI: 10.1146/annurev.ns.33.120183.001543.

Singleterry, R.C., S.R. Blattnig, M.S. Clowdsley, G.D. Qualls, C.A. Sandridge, et al. OLTARIS: On-line tool for the assessment of radiation in space. Acta Astronaut., 68, 1086-1097, 2011, DOI: $10.1016 /$ j.actaastro.2010.09.022.

Slaba, T.C., and S.R. Blattnig. GCR environmental models I: sensitivity analysis for GCR environments. Space Weather, 12 (4), 217-224, 2014, DOI: 10.1002/2013sw001025.

Slaba, T.C., S.R. Blattnig, S.K. Aghara, L.W. Townsend, T. Handler, T.A. Gabriel, L.S. Pinsky, and B. Reddell. Coupled neutron transport for HZETRN. Radiat. Meas., 45, 173-182, 2010a, DOI: $10.1016 /$ j.radmeas.2010.01.005.

Slaba, T.C., S.R. Blattnig, and F.F. Badavi. Faster and more accurate transport procedures for HZETRN. J. Comput. Phys., 229, 9397-9417, 2010b, DOI: 10.1016/j.jcp.2010.09.010.

Slaba, T.C., S.R. Blattnig, and J. Tweed. Reduced discretization error in HZETRN. J. Comput. Phys., 234, 217-229, 2013, DOI: $10.1016 /$ j.jcp.2012.09.042.

Slaba, T.C., X.J. Xu, S.R. Blattnig, and R.B. Norman. GCR environmental models III: GCR model validation and propagated uncertainties in effective dose. Space Weather, 12 (4), 233-245, 2014, DOI: 10.1002/2013sw001027.

Smith, D.E., M.T. Zuber, H.V. Frey, J.B. Garvin, J.W. Head, et al. Mars Orbiter Laser Altimeter: experiment summary after the first year of global mapping of Mars. J. Geophys. Res. [Planets], 106 (E10), 23689-23722, 2001, DOI: $10.1029 / 2000 J E 001364$.

Townsend, L.W., M. PourArsalan, F.A. Cucinotta, M.Y. Kim, and N.A. Schwadron. Transmission of galactic cosmic rays through 
Mars atmosphere. Space Weather, 9, S00E11, 2011, DOI: 10.1029/2009sw000564.

Usoskin, I.G., G.A. Bazilevskaya, and G.A. Kovaltsov. Solar modulation parameter for cosmic rays since 1936 reconstructed from ground-based neutron monitors and ionization chambers. J. Geophys. Res. [Space Phys.], 116 (A2), A02104, 2011, DOI: 10.1029/2010JA016105.

Wilson, J.W., L.W. Townsend, J.E. Nealy, S.Y. Chun, B.S. Hong, W.W. Buck, S.L. Lamkin, B.D. Ganapol, F. Khan, and F.A. Cucinotta. BRYNTRN: a baryon transport model. NASA STI/ Recon Technical Report N, 89, 17562, 1989.

Wilson, J.W., L. Townsend, W. Schimmerling, G.S. Khandelwal, F. Khan, J.E. Nealy, F.A. Cucinotta, L.C. Simonsen, J.L. Shinn, and J.W. Norbury. Transport methods and interactions for space radiations. Report NASA RP 1257, NASA, 1991.
Wilson, J.W., T.C. Slaba, F.F. Badavi, B.D. Reddell, and A.A. Bahadori. Advances in NASA radiation transport research: 3DHZETRN. Life Sciences in Space Research, 2, 6-22, 2014, DOI: 10.1016/j.lssr.2014.05.003.

Wilson, J.W., T.C. Slaba, F.F. Badavi, B.D. Reddell, and A.A. Bahadori. 3DHZETRN: shielded ICRU spherical phantom. Life Sciences in Space Research, 4, 46-61, 2015, DOI: 10.1016/j.1ssr.2015.01.002.

Zeitlin, C., D.M. Hassler, F.A. Cucinotta, B. Ehresmann, R.F. Wimmer-Schweingruber, et al. Measurements of energetic particle radiation in transit to Mars on the Mars Science Laboratory. Science, 340, 1080-1084, 2013, DOI: $10.1126 /$ science. 1235989 .

Cite this article as: Matthiä D, Ehresmann B, Lohf H, Köhler J, Zeitlin C, et al. The Martian surface radiation environment - a comparison of models and MSL/RAD measurements. J. Space Weather Space Clim., 6, A13, 2016, DOI: 10.1051/swsc/2016008. 